\title{
Repetition threshold for circular words
}

\author{
Irina A. Gorbunova \\ Ural Federal University \\ Ekaterinburg, Russia \\ i.a.gorbunova@gmail.com
}

\author{
Submitted: May 19, 2012; Accepted: Oct 13, 2012; Published: Oct 25, 2012 \\ Mathematics Subject Classifications: 68R15
}

\begin{abstract}
We find the threshold between avoidable and unavoidable repetitions in circular words over $k$ letters for any $k \geqslant 6$. Namely, we show that the number $\operatorname{CRT}(k)=$ $\frac{\lceil k / 2\rceil+1}{\mid k / 2\rceil}$ satisfies the following properties. For any $n$ there exists a $k$-ary circular word of length $n$ containing no repetition of exponent greater than $\operatorname{CRT}(k)$. On the other hand, $k$-ary circular words of some lengths must have a repetition of exponent at least $\operatorname{CRT}(k)$.
\end{abstract}

Keywords: Dejean's conjecture, repetition threshold, circular words

\section{Introduction}

Families of combinatorial objects parametrized by a certain numerical parameter are ubiquitous not only in combinatorics, but all over mathematics and elsewhere. One of the most interesting phenomena that may happen with such family, when their properties sharply change at certain value of the parameter, is often called phase transition or threshold value. Discovering and evaluating threshold values is an important and challenging task, as they often depend on deep properties of the objects in question. This paper concerns the threshold value phenomenon in combinatorics of words, where it occurs when we consider sets of words with forbidden repetitions.

Recall that the exponent of a word $w$ is the ratio between its length and its minimal period: $\exp (w)=|w| / \operatorname{per}(w)$. If this ratio is greater than 1 , then $w$ is a (fractional) power. A word is called $\beta$-free for some rational $\beta>1$ if the exponent of any its factor is less than $\beta$. The repetition threshold function $\mathrm{RT}(k)$ was introduced by Dejean [4]. The celebrated Dejean's conjecture, recently completely settled, see $[2-4,7]$, states that there are infinitely many $k$-ary $\beta$-free words if and only if $\beta>\mathrm{RT}(k)$, where

\begin{tabular}{|c|c|c|c|c|c|c|c|c|}
\hline$k$ & 2 & 3 & 4 & 5 & 6 & $\cdots$ & $m$ & $\cdots$ \\
\hline $\operatorname{RT}(k)$ & 2 & $7 / 4$ & $7 / 5$ & $5 / 4$ & $6 / 5$ & $\cdots$ & $m /(m-1)$ & $\cdots$ \\
\hline
\end{tabular}


Dejean's conjecture attracted interest to the area and gave rise to a number of similar "threshold" problems. For example, Ilie, Ochem, and Shallit [6] introduced the notion of generalized repetition threshold $\mathrm{RT}(k, l)$ to handle avoidance of repetitions with periods $\geqslant l$ only. The best lower bound for $\mathrm{RT}(k, l)$ was given in [5]: for any $k \geqslant 3, l \geqslant 3$,

$$
\mathrm{RT}(k, l) \geqslant 1+\frac{1}{(5 l / 6-1 / 2+O(1 / l)) k} .
$$

The best upper bound for $\operatorname{RT}(k, l)$ was presented in [8]: for any $k \geqslant 2, l \geqslant 1$, and for some constant $c$,

$$
\mathrm{RT}(k, l) \leqslant 1+\frac{c}{l k} .
$$

Samsonov and Shur [9] considered Abelian powers of words instead of the usual powers and obtained the lower bounds for Abelian repetition threshold: $\operatorname{ART}_{S}(k) \geqslant(k-2) /(k-3)$ for all $k \geqslant 5 ; \operatorname{ART}_{W}(k) \geqslant k /(k-2)$ for all $k \geqslant 10$ (the subscripts here stand for "strong" and "weak" Abelian fractional powers). In this paper we are interested in the repetition threshold for circular words.

Circular words form an obvious variation of ordinary words: we just link up the ends of a word, thus getting a cyclic sequence of letters without beginning or end. The circular word obtained from a word $w$ will be denoted by $(w)$, e.g.,

$$
w=a b c d a c b \quad \begin{array}{llll}
a & b \\
c & (w) & c \\
\hline a & d & d \\
\hline
\end{array}
$$

A circular word can also be viewed as the representation of a conjugacy class of ordinary words. Two words $u$ and $v$ are conjugates if there are words $x$ and $y$ such that $u=x y$ and $v=y x$. Clearly, conjugacy is an equivalence relation, and the conjugacy class of a word $w$ can be identified with the circular word $(w)$.

Avoidance properties of a word are inherited by all its factors. Hence, any set of words defined by forbidden repetitions is closed under taking factors. But circular words cannot inherit properties in a similar way, because the factors of a circular word are ordinary, not circular, words. As a result, there are three natural definitions of the repetition threshold that are equivalent for words but not for circular words. Namely, RT $(k)$ can be defined as the infimum of the set of all $\beta$ such that

- there are arbitrarily long $k$-ary $\beta$-free words;

- there are $k$-ary $\beta$-free words of any length except for a finite set;

- there are $k$-ary $\beta$-free words of any length.

For circular words, these conditions define three different functions: weak repetition threshold $\mathrm{CRT}_{W}(k)$, intermediate repetition threshold $\mathrm{CRT}_{I}(k)$, and strong repetition threshold $\mathrm{CRT}_{S}(k)$, respectively.

In this paper, we deal with strong repetition threshold only and write $\operatorname{CRT}(k)$ for it. It is known that $\operatorname{CRT}(2)=5 / 2$ (see [1]) and CRT(3) $=2$ (this follows, e.g., from 
the results of $[10])$. The aim of this paper is to prove the formula $\operatorname{CRT}(k)=\frac{\lceil k / 2\rceil+1}{\lceil k / 2\rceil}$ for all $k \geqslant 6$. The lower bound (Proposition 1) is almost trivial. For the upper bound, we explicitly construct $k$-ary circular words of every length that are $\beta$-free for all $\beta>\frac{\lceil k / 2\rceil+1}{\lceil k / 2\rceil}$. Four different constructions cover the cases odd $k \geqslant 11, k=6, k=7$, and $k=9$ (Theorems 2,4,5,6). Then the result for the remaining even values of $k$ follows trivially.

\section{Circular repetition threshold for large alphabets}

We say that a word is $\beta^{+}$-free if it is $\beta^{\prime}$-free for all $\beta^{\prime}>\beta$.

Proposition 1. For any $k \geqslant 4$, there are no $k$-ary circular $\frac{\lceil k / 2\rceil+1}{\lceil k / 2\rceil}$-free words of length $k+1$.

Proof. Take a circular word $(w)$ of length $k+1$ over $k$ letters. By the Pigeonhole Principle, there is a letter $x$ that appears in $(w)$ at least twice. Hence $(w)=\left(x w_{1} x w_{2}\right)$. Since $\min \left\{\left|w_{1}\right|,\left|w_{2}\right|\right\} \leqslant(k-1) / 2<\lceil k / 2\rceil$, we obtain $\max \left\{\exp \left(x w_{1} x\right), \exp \left(x w_{2} x\right)\right\} \geqslant \frac{\lceil k / 2\rceil+1}{\lceil k / 2\rceil}$. The proposition is proved.

From Proposition 1 it follows that $\mathrm{CRT}(k) \geqslant \frac{\lceil k / 2\rceil+1}{\lceil k / 2\rceil}$.

Theorem 2. For any odd $k \geqslant 11$, there exist $k$-ary circular $\left(\frac{\lceil k / 2\rceil+1}{\lceil k / 2\rceil}\right)^{+}$-free words of any length.

Proof. We write $w[i]$ for the $i$ th letter of $w$ and $\overleftarrow{w}$ for the reversal of $w$. Let $r=\lceil k / 2\rceil$ For any positive integer $n$ we construct a circular $((r+1) / r)^{+}$-free word $(w)$ of length $n$ over the alphabet $\{1, \ldots, k\}$. For $n \leqslant k$, it is trivial. We first assume $n \geqslant 2 k+6$ and consider the case $k+1 \leqslant n \leqslant 2 k+5$ later.

We construct the circular word $(w)$ of the form $w=L_{1} u L_{2} v$, where

- $u$ is a $((r+1) / r)^{+}$-free word of length $\lceil(n-4) / 2\rceil$ over the alphabet $A=\{1, \ldots, r+1\}$ and $u[1]=u[r+1]$;

- $v$ is a $((r+1) / r)^{+}$-free word of length $\lfloor(n-4) / 2\rfloor$ over the alphabet $B=\{r-1, \ldots, k\}$;

- $L_{1}=a_{i_{0}} b_{i_{0}}$ such that $a_{i_{0}} \in A \backslash B, b_{i_{0}} \in B \backslash A$;

- $L_{2}=b_{j_{0}} a_{j_{0}}$ such that $a_{j_{0}} \in A \backslash B, b_{j_{0}} \in B \backslash A$.

Observation 3. If $u$ or $v$ has a factor ptp, then $\exp (p t p) \leqslant((r+1) / r)$ by the choice of $u$ and $v$. If in this case $(w)=\left(p t p t^{\prime}\right)$, then $\left|p t^{\prime} p\right|>|p t p|$. Thus, $\exp \left(p t^{\prime} p\right)<(r+1) / r$. Therefore, if $(w)$ has a factor ptp of forbidden exponent, then either one of these occurrences is inside $u$ and the other one is inside $v$, or at least one of these occurrences intersects $L_{1}$ or $L_{2}$. In the first case, $|p| \leqslant 3$, because the alphabets of $u$ and $v$ have only three common letters. In the second case, $p$ is also short due to the structure of $L_{1}$ and $L_{2}$. 
The $(r+1)$-ary $((r+1) / r)^{+}$-free words of any length exist by Dejean's conjecture. Moreover, an infinite $(r+1)$-ary $((r+1) / r)^{+}$-free word $W$ certainly contains a factor of the form $a_{1} a_{2} \cdots a_{r} a_{1}$. This factor can be extended to the right up to the length $\lceil(n-4) / 2\rceil$. So we take this extension as $u$ to ensure that $u[1]=u[r+1]$.

Denote the common letters of $A$ and $B$ by $x, y$, and $z$. If $n$ is even, we put $v=f(u)$, where $f: A \rightarrow B$ is any bijection such that $f(x)=x, f(y)=y$, and $f(z)=z$. If $n$ is odd, we take the word $f(u)$ without the first letter as $v$.

By $u_{1}=a_{i_{1}} \cdots a_{i_{r}}$ (resp., $v_{1}=b_{j_{1}} \cdots b_{j_{r}}$ ) we denote the prefix of $u$ (resp., $v$ ) of the length $r$. The suffixes of $u$ and $v$ of length $r$ are denoted by $u_{2}=a_{j_{r}} \cdots a_{j_{1}}$ and $v_{2}=b_{i_{r}} \cdots b_{i_{1}}$. Since $|u|,|v| \geqslant 2 r$, we see that these prefixes and suffixes do not overlap. Then $(w)$ looks like in Fig. 1.

Obviously, all letters in $u_{1}$ [resp., in $u_{2}, v_{1}$, and $v_{2}$ ] are different. Let $\Sigma(t)$ be a set of all letters of the word $t$. Then either (Case I) $\Sigma\left(u_{1}\right)=\Sigma\left(u_{2}\right)$ and $\Sigma\left(v_{1}\right)=\Sigma\left(v_{2}\right)$ or (Case II) $\Sigma\left(u_{1}\right)$ and $\Sigma\left(u_{2}\right)$ [resp., $\Sigma\left(v_{1}\right)$ and $\left.\Sigma\left(v_{2}\right)\right]$ differ by one letter.

We proceed as follows. First, we describe, for both Case I and Case II, some transformations of the word $(w)$ (for Case II, we need to consider 25 subcases based on the distribution of letters in $u)$. Then we prove that the resulting circular word is $((r+1) / r)^{+}$free in all cases.

Case I. To make the constructed word $(w)((r+1) / r)^{+}$-free, we perform a certain permutation of letters in $u$ and $v$. Each permutation on $A$ induces a unique permutation on $B$ preserving the equality $v=f(u)$. Suppose $\hat{a} \in A$ is the letter that is not in $\Sigma\left(u_{1}\right)$; then we switch $\hat{a}$ and $z$. To each letter $a \in \Sigma\left(u_{1}\right)$, we assign a pair of positions $g(a)=\left(s_{1}, s_{2}\right)$ such that $a_{i_{s_{1}}}=a_{j_{s_{2}}}=a$. There are at least $(r-3) \geqslant 3$ pairs that are not of the form: $(1,-),(-, 1),(-, r)$. Among these pairs, choose the pair $\left(\tilde{s}_{1}, \tilde{s}_{2}\right)$ with the biggest first coordinate and switch $g^{-1}\left(\tilde{s}_{1}, \tilde{s}_{2}\right)$ and $x$. Note that $\tilde{s}_{1} \in\{r-2, r-1, r\}$. Let $\left(\hat{s}_{1}, \hat{s}_{2}\right)$ be another one of these pairs such that $\hat{s}_{1}<\tilde{s}_{1}-1$ (for instance, one can take the pair with the least first coordinate). Then we switch $g^{-1}\left(\hat{s}_{1}, \hat{s}_{2}\right)$ and $y$. Finally, we switch $y$ and $z$ in the word $v$ only.

The words $L_{1}$ and $L_{2}$ are defined by the conditions $a_{j_{0}}=a_{j_{r}}, a_{i_{0}} \in\left\{a_{i_{r-1}}, a_{i_{r}}\right\} \backslash\{x\}$, $b_{i_{0}}=b_{i_{r}}, b_{j_{0}} \in\left\{b_{j_{r-1}}, b_{j_{r}}\right\} \backslash\{x\}$ for $n$ even (see Fig. 1, left), $b_{j_{0}} \in\left\{b_{j_{1}}, b_{j_{r}}\right\} \backslash\{x\}$ for $n$ odd (see Fig. 1, right).

Case II. Let $a_{i_{s_{1}}}=\Sigma\left(u_{1}\right) \backslash \Sigma\left(u_{2}\right)$ and $a_{j_{s_{2}}}=\Sigma\left(u_{2}\right) \backslash \Sigma\left(u_{1}\right)$. Switch $a_{i_{s_{1}}}$ and $y ; a_{j_{s_{2}}}$ and $z$. The letter that should be switched with $x$, depends on $s_{1}$ and $s_{2}$ : 25 cases are to be studied. The large number of different cases is due to the fact that $u[1]=u[r+1]$, while no symmetric condition for the end of $u$ can be assumed. For each case, we consider separately the upper and the lower part of the circle (the words $v_{2} L_{1} u_{1}$ and $u_{2} L_{2} v_{1}$, respectively; for better readability, $u_{2} L_{2} v_{1}$ is reversed). In some cases, these words are constructed separately for even and odd lengths of the whole word $(w)$. In each case, $x$ can be switched with any letter $a$ such that $a$ occurs in the right of two factors depicted with underbraces, and $f(a)$ occurs in the left of these factors. At least one such letter $a$ exists, because the total length of depicted factors is at least $r$ and these factors contain neither $y$ nor $z$. The additional switches for $v$, if any, are mentioned explicitly. The words $L_{1}=a_{i_{0}} b_{i_{0}}$ and $L_{2}=b_{j_{0}} a_{j_{0}}$ are chosen such that (1) $a_{i_{0}} \in\left\{a_{i_{r-1}}, a_{i_{r}}\right\}, a_{j_{0}} \in$ 

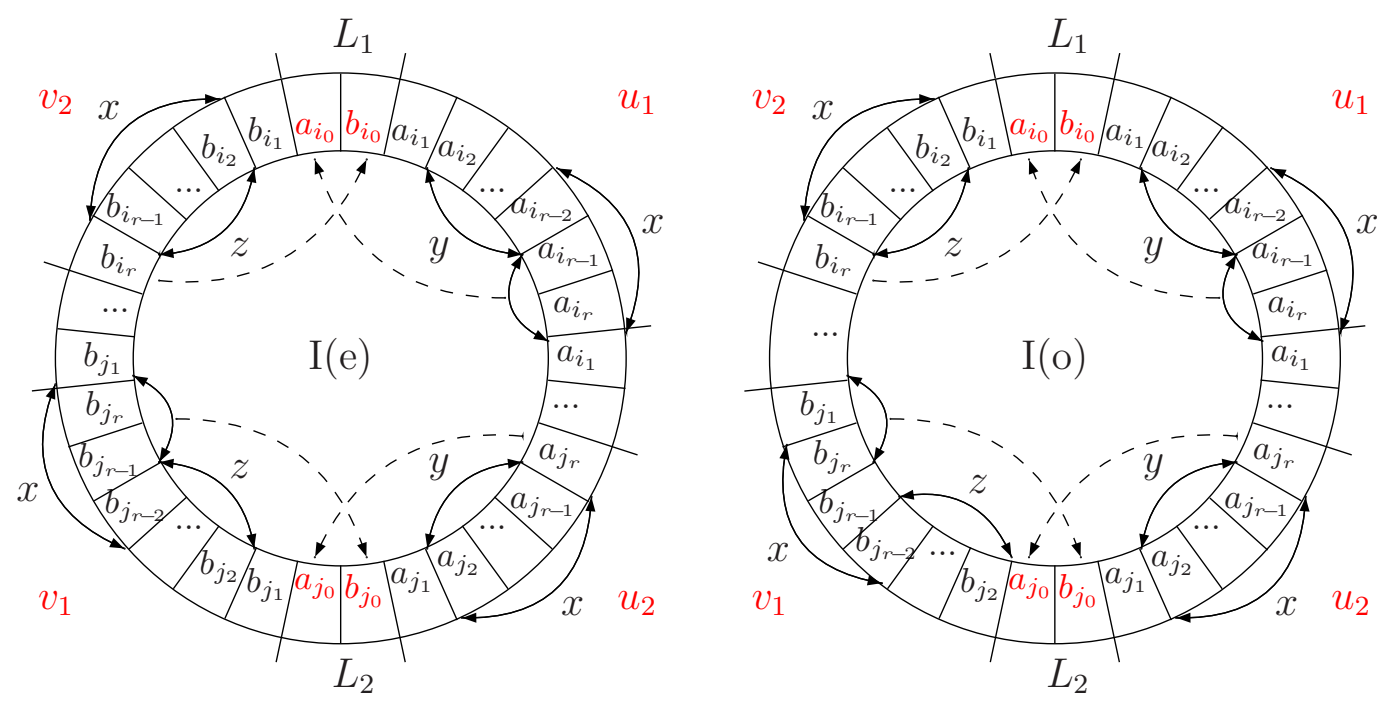

Figure 1: The circular word $(w)$ of length $n$ : Case I for even $n$ (left) and for odd $n$ (right).

$\left\{a_{j_{r-1}}, a_{j_{r}}\right\}, b_{i_{0}} \in\left\{b_{i_{r-1}}, b_{i_{r}}\right\}, b_{j_{0}} \in\left\{b_{j_{r-1}}, b_{j_{r}}\right\}$ if $n$ is even, and $b_{j_{0}} \in\left\{b_{j_{1}}, b_{j_{r}}\right\}$ if $n$ is odd; (2) $\left\{a_{i_{0}}, a_{j_{0}}, b_{i_{0}}, b_{j_{0}}\right\} \cap\{x, y, z\}=\varnothing$; (3) if possible under conditions (1), (2), then $a_{i_{0}} \neq a_{j_{0}}, b_{i_{0}} \neq b_{j_{0}}$. Note that $x, y, z$ are chosen so that (1),(2) certainly can be satisfied.

Case 1: $\left(s_{1}, s_{2}\right)=(1,1)$.

$v_{2} L_{1} u_{1} \quad: \quad b_{i_{r}} \underbrace{b_{i_{r-1}} \quad \cdots}_{x} \quad b_{i_{3}} b_{i_{2}} z \quad a_{i_{0}} b_{i_{0}} \quad y a_{i_{2}} \cdots \underbrace{a_{i_{r-3}} a_{i_{r-2}} a_{i_{r-1}}}_{x} a_{i_{r}}$

$\overleftarrow{u_{2} L_{2} v_{1}}(e): \quad b_{j_{r}} \underbrace{b_{j_{r-1}} b_{j_{r-2}} b_{j_{r-3}}}_{x} \cdots b_{j_{2}} y \quad a_{j_{0}} b_{j_{0}} z a_{j_{2}} \underbrace{a_{j_{3}} \ldots a_{j_{r-1}}}_{x} a_{j_{r}}$

$\overleftarrow{u_{2} L_{2} v_{1}}(o): \quad y b_{j_{r}} \underbrace{b_{j_{r-1} b_{j_{r-2}} b_{j_{r-3}}}^{x}}_{x} \cdots b_{j_{2}} a_{j_{0}} b_{j_{0}} z a_{j_{2}} \underbrace{a_{j_{3}} \quad \cdots \quad a_{j_{r-1}}}_{x} a_{j_{r}}$

Case 2: $\left(s_{1}, s_{2}\right)=(2,2)$.

$v_{2} L_{1} u_{1}(e): \quad b_{i_{r}} b_{i_{r-1}} \underbrace{b_{i_{r-2}} \cdots b_{i_{3}}}_{x} z b_{i_{1}} \quad a_{i_{0}} b_{i_{0}} \quad a_{i_{1}} y \cdots \underbrace{a_{i_{r-3}} a_{i_{r-2}} a_{i_{r-1}} a_{i_{r}}}_{x}\left[a_{i_{1}}\right]$

$\overleftarrow{u_{2} L_{2} v_{1}}(e):\left[b_{j_{1}}\right] \underbrace{b_{j_{r}} b_{j_{r-1}} b_{j_{r-2}} b_{j_{r-3}}}_{x} \cdots y b_{j_{1}} a_{j_{0}} b_{j_{0}} \quad a_{j_{1}} z \underbrace{a_{j_{3}} \cdots a_{j_{r-2}}}_{x} a_{j_{r-1}} a_{j_{r}}$

If $n$ is odd, then to avoid the repetition $x y a_{j_{0}} \cdots x y a_{j_{0}}$ we choose new position for $x$ in $v$. We also require $a_{j_{0}} \in\left\{a_{j_{r-1}}, a_{j_{r}}\right\} \backslash\left\{a_{i_{1}}\right\}$.

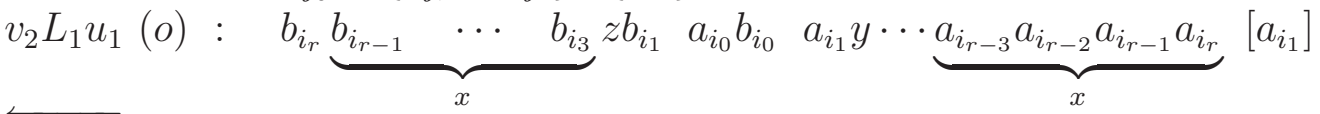

$$
\begin{aligned}
& \overleftarrow{u_{2} L_{2} v_{1}}(o): \quad b_{j_{1}} \underbrace{b_{j_{r}} b_{j_{r-1}} b_{j_{r-2}}}_{x} \cdots b_{j_{3}} y a_{j_{0}} b_{j_{0}} \quad a_{j_{1}} z \underbrace{a_{j_{3}} \cdots a_{j_{r-2}}}_{x} a_{j_{r-1}} a_{j_{r}}
\end{aligned}
$$

Case 3: $\left(s_{1}, s_{2}\right) \in\{3, \ldots, r-2\} \times\{3, \ldots, r-2\}$. In $v, z$ and $x$ are switched. 


$$
\begin{aligned}
& v_{2} L_{1} u_{1} \quad: \quad b_{i_{r}} b_{i_{r-1}} \underbrace{\overbrace{b_{i_{r-2}} \cdots b_{i_{3}}}^{x} b_{i_{2}} b_{i_{1}}}_{z} \quad a_{i_{0}} b_{i_{0}} a_{i_{1}} a_{i_{2}} \overbrace{a_{i_{3}} \cdots \underbrace{a_{i_{r-3}} a_{i_{r-2}}}_{x} a_{i_{r-1}} a_{i_{r}}}^{y}\left[a_{i_{1}}\right] \\
& \overleftarrow{u_{2} L_{2} v_{1}}(e):\left[b_{j_{1}}\right] \underbrace{b_{j_{r}} b_{j_{r-1}} \overbrace{b_{j_{r-2}} b_{j_{r-3}}}^{y} \cdots b_{j_{3}}}_{z} b_{j_{2}} b_{j_{1}} a_{j_{0}} b_{j_{0}} \underbrace{a_{j_{1}} a_{j_{2}} \overbrace{a_{j_{3}} \cdots a_{j_{r-2}}}^{z}}_{x} a_{j_{r-1}} a_{j_{r}} \\
& \overleftarrow{u_{2} L_{2} v_{1}}(o): \quad b_{j_{1}} \underbrace{b_{j_{r}} b_{j_{r-1}} \overbrace{b_{j_{r-2}} b_{j_{r-3}}}^{y} \cdots b_{j_{3}}}_{z} b_{j_{2}} a_{j_{0}} b_{j_{0}} \underbrace{a_{j_{1}} a_{j_{2}} \overbrace{a_{j_{3}} \cdots a_{j_{r-2}}}^{z}}_{x} a_{j_{r-1}} a_{j_{r}}
\end{aligned}
$$

Case 4: $\left(s_{1}, s_{2}\right)=(r-1, r-1)$. In $v, z$ and $x$ are switched.

$v_{2} L_{1} u_{1} \quad: \quad b_{i_{r}} x \underbrace{b_{i_{r-2}} \cdots b_{i_{2}}}_{z} b_{i_{1}} a_{i_{0}} b_{i_{0}} \quad a_{i_{1}} \underbrace{a_{i_{2}} \cdots a_{i_{r-2}}}_{x} y a_{i_{r}}$

$\overleftarrow{u_{2} L_{2} v_{1}}(e): \quad b_{j_{r}} y \underbrace{b_{j_{r-2}} \cdots b_{j_{2}}}_{z} b_{j_{1}} a_{j_{0}} b_{j_{0}} \quad a_{j_{1}} \underbrace{a_{j_{2}} \cdots a_{j_{r-2}}}_{x} z a_{j_{r}}$

$\overleftarrow{u_{2} L_{2} v_{1}}(o): \quad b_{j_{1}} b_{j_{r}} y \underbrace{b_{j_{r-2}} \cdots b_{j_{2}}}_{z} a_{j_{0}} b_{j_{0}} \quad a_{j_{1}} \underbrace{a_{j_{2}} \cdots a_{j_{r-2}}}_{x} z a_{j_{r}}$

Case 5: $\left(s_{1}, s_{2}\right)=(r, r)$. In $v, y$ and $x$ are switched.

$v_{2} L_{1} u_{1} \quad: \quad z b_{i_{r-1}} \underbrace{b_{i_{r-2}} \cdots b_{i_{2}}}_{y} b_{i_{1}} \quad a_{i_{0}} b_{i_{0}} \quad a_{i_{1}} \underbrace{a_{i_{2}} \cdots a_{i_{r-2}}}_{x} a_{i_{r-1}} y\left[a_{i_{1}}\right]$

$\overleftarrow{u_{2} L_{2} v_{1}}(e):\left[b_{j_{1}}\right] x b_{j_{r-1}} \underbrace{b_{j_{r-2}} \cdots b_{j_{2}}}_{y} b_{j_{1}} a_{j_{0}} b_{j_{0}} a_{j_{1}} \underbrace{a_{j_{2}} \cdots a_{j_{r-2}}}_{x} a_{j_{r-1}} z$

$\overleftarrow{u_{2} L_{2} v_{1}}(o): \quad b_{j_{1}} x b_{j_{r-1}} \underbrace{b_{j_{r-2}} \cdots b_{j_{2}}}_{y} a_{j_{0}} b_{j_{0}} a_{j_{1}} \underbrace{a_{j_{2}} \cdots a_{j_{r-2}}}_{x} a_{j_{r-1}} z$

Case 6: $\left(s_{1}, s_{2}\right)=(1,2)$.

$v_{2} L_{1} u_{1} \quad: \quad b_{i_{r}} \underbrace{b_{i_{r-1}} \quad \cdots}_{x} \quad b_{i_{3}} z b_{i_{1}} \quad a_{i_{0}} b_{i_{0}} \quad y a_{i_{2}} \cdots \underbrace{a_{i_{r-3}} a_{i_{r-2}} a_{i_{r-1}}}_{x} a_{i_{r}}$

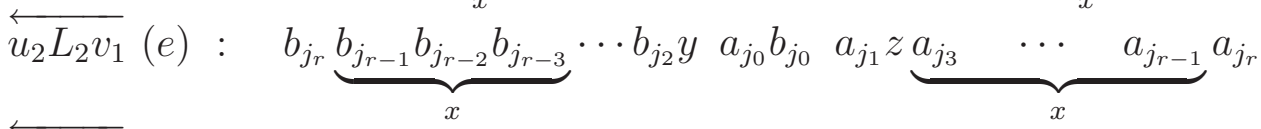

$\overleftarrow{u_{2} L_{2} v_{1}}(o): \quad y b_{j_{r}} \underbrace{b_{j_{r-1}} b_{j_{r-2}} b_{j_{r-3}}}_{x} \cdots b_{j_{2}} a_{j_{0}} b_{j_{0}} \quad a_{j_{1}} z \underbrace{a_{j_{3}} \quad \cdots \quad a_{j_{r-1}}}_{x} a_{j_{r}}$

Case 7: $\left(s_{1}, s_{2}\right)=(2,1)$ is symmetric to Case 6 . This means that if we reverse $u$ and $v$, we will get Case 6 . (If $n$ is odd, we delete the first letter of $\overleftarrow{v}$, i.e., $b_{i_{1}}$.)

Case 8: $\left(s_{1}, s_{2}\right) \in\{1\} \times\{3, \ldots, r-2\}$. In $v, z$ and $x$ are switched. $v_{2} L_{1} u_{1} \quad: \quad b_{i_{r}} b_{i_{r-1}} \underbrace{\overbrace{b_{i_{r-2}} \cdots b_{i_{3}}}^{x} b_{i_{2}} b_{i_{1}}}_{z} a_{i_{0}} b_{i_{0}} \quad y a_{i_{2}} \cdots \underbrace{a_{i_{r-3}} a_{i_{r-2}} a_{i_{r-1}}}_{x} a_{i_{r}}$ $\overleftarrow{u_{2} L_{2} v_{1}}(e): \quad b_{j_{r}} \underbrace{b_{j_{r-1}} b_{j_{r-2}} b_{j_{r-3}}}_{z} \cdots b_{j_{2}} y \quad a_{j_{0}} b_{j_{0}} \underbrace{a_{j_{1}} a_{j_{2}} \overbrace{a_{j_{3}} \cdots a_{j_{r-2}}}^{z}}_{x} a_{j_{r-1}} a_{j_{r}}$ $\overleftarrow{u_{2} L_{2} v_{1}}(o): \quad y b_{j_{r}} \underbrace{b_{j_{r-1}} b_{j_{r-2}} b_{j_{r-3}}}_{z} \cdots b_{j_{2}} \quad a_{j_{0}} b_{j_{0}} \underbrace{a_{j_{1}} a_{j_{2}} \overbrace{a_{j_{3}} \cdots a_{j_{r-2}}}^{z}}_{x} a_{j_{r-1}} a_{j_{r}}$ 
Case 9: $\left(s_{1}, s_{2}\right) \in\{3, \ldots, r-2\} \times\{1\}$. If $n$ is even, then this case is symmetric to Case 8. If $n$ is odd, then we choose new position for $x$ and switch $y$ and $x$ in $v$.

$$
\begin{aligned}
& v_{2} L_{1} u_{1}(o): \quad b_{i_{r}} \underbrace{b_{i_{r-1}} b_{i_{r-2}} b_{i_{r-3}}}_{y} \cdots b_{i_{2}} z \quad a_{i_{0}} b_{i_{0}} \quad a_{i_{1}} a_{i_{2}} \underbrace{\overbrace{a_{i_{3}} \cdots a_{i_{r-2}}} a_{i_{r-1}} a_{i_{r}}}_{x}\left[a_{i_{1}}\right] \\
& \overleftarrow{u_{2} L_{2} v_{1}}(o): \quad b_{j_{1}} \underbrace{b_{j_{r}} b_{j_{r-1}} \overbrace{b_{j_{r-2}} \cdots b_{j_{3}}}^{x}}_{y} b_{j_{2}} \quad a_{j_{0}} b_{j_{0}} \quad z a_{j_{2}} \cdots \underbrace{a_{j_{r-3}} a_{j_{r-2}} a_{j_{r-1}}}_{x} a_{j_{r}}
\end{aligned}
$$

Case 10: $\left(s_{1}, s_{2}\right)=(1, r-1)$. In $v, z$ and $x$ are switched.

$v_{2} L_{1} u_{1} \quad: \quad b_{i_{r}} x \underbrace{b_{i_{r-2}} \cdots b_{i_{2}}}_{z} b_{i_{1}} \quad a_{i_{0}} b_{i_{0}} \quad y a_{i_{2}} \underbrace{a_{i_{3}} \cdots a_{i_{r-1}}}_{x} a_{i_{r}}$

$\overleftarrow{u_{2} L_{2} v_{1}}(e): \quad b_{j_{r}} \underbrace{b_{j_{r-1} \cdots b_{j_{3}}}}_{z} b_{j_{2}} y a_{j_{0}} b_{j_{0}} \quad a_{j_{1}} \underbrace{a_{j_{2}} \cdots a_{j_{r-2}}}_{x} z a_{j_{r}}$

$\overleftarrow{u_{2} L_{2} v_{1}}(o): \quad y b_{j_{r}} \underbrace{b_{j_{r-1}} \cdots b_{j_{3}}}_{z} b_{j_{2}} \quad a_{j_{0}} b_{j_{0}} \quad a_{j_{1}} \underbrace{a_{j_{2}} \cdots a_{j_{r-2}}}_{x} z a_{j_{r}}$

Case 11: $\left(s_{1}, s_{2}\right)=(r-1,1)$ is symmetric to Case 10.

Case 12: $\left(s_{1}, s_{2}\right)=(1, r)$.

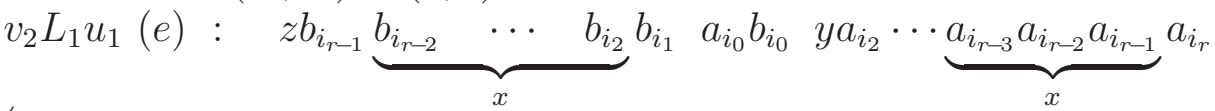

$\overleftarrow{u_{2} L_{2} v_{1}}(e): \quad b_{j_{r}} \underbrace{b_{j_{r-1}} b_{j_{r-2}} b_{j_{r-3}}}_{x} \cdots b_{j_{2}} y \quad a_{j_{0}} b_{j_{0}} a_{j_{1}} \underbrace{a_{j_{2}} \cdots a_{j_{r-2}}}_{x} a_{j_{r-1}} z$

Here $\bar{x} \in A \backslash\{x\}$ and $\bar{z} \in B \backslash\{z\}$. In $v, z$ and $x$ are switched.

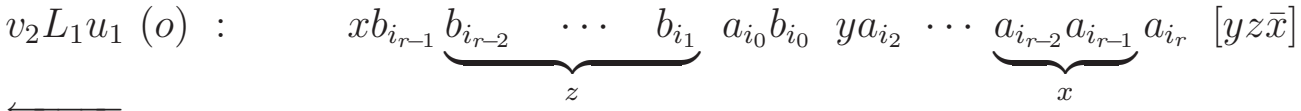

$$
\begin{aligned}
& \overleftarrow{u_{2} L_{2} v_{1}}(o):[\bar{z} x] \quad y b_{j_{r}} \underbrace{b_{j_{r-1}} b_{j_{r-2}}}_{z} \cdots b_{j_{2}} a_{j_{0}} b_{j_{0}} \underbrace{a_{j_{1}} \cdots a_{j_{r-2}}}_{x} a_{j_{r-1}} z
\end{aligned}
$$

Case 13: $\left(s_{1}, s_{2}\right)=(r, 1)$ is symmetric to Case 12 (if $n$ is odd, then we switch $z$ and $x$ in $v)$.

Case 14: $\left(s_{1}, s_{2}\right) \in\{2\} \times\{3, \ldots, r-2\}$. In $v, z$ and $x$ are switched.

$$
\begin{aligned}
& v_{2} L_{1} u_{1} \quad: \underbrace{b_{i_{r}} b_{i_{r-1}} \overbrace{b_{i_{r-2}} \cdots b_{i_{3}}} b_{i_{2}} b_{i_{1}}}_{z} a_{i_{0}} b_{i_{0}} \quad a_{i_{1}} y a_{i_{3}} \quad \cdots \quad x a_{i_{r-1}} a_{i_{r}}
\end{aligned}
$$

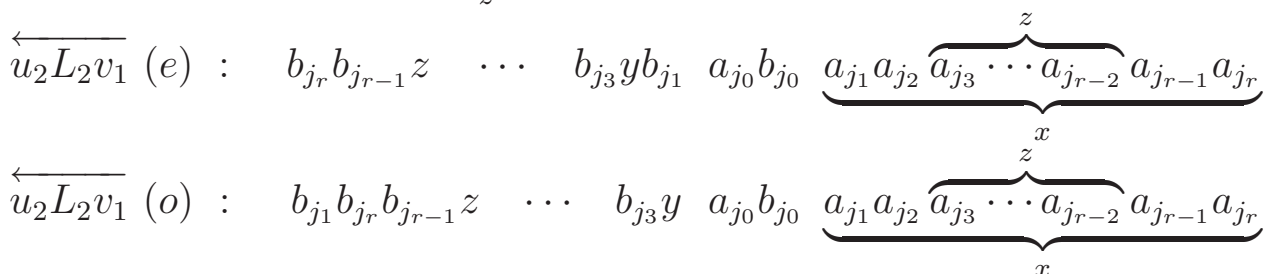

Case 15: $\left(s_{1}, s_{2}\right) \in\{3, \ldots, r-2\} \times\{2\}$. If $n$ is even, then this case is symmetric to Case 14. For odd $n$, we switch $x$ and $y$ in $v$. To avoid the repetition $x y a_{j_{0}} \cdots x y a_{j_{0}}$ we choose new position for $y$ in $v$. 
$v_{2} L_{1} u_{1}(o): \quad b_{i_{r}} b_{i_{r-1}} \underbrace{b_{i_{r-2}} b_{i_{r-3}}}_{y} \cdots z b_{i_{1}} \quad a_{i_{0}} b_{i_{0}} \quad a_{i_{1}} \underbrace{a_{i_{2}} \overbrace{a_{i_{3}} \cdots a_{i_{r-2}}}^{y} a_{i_{r-1}} a_{i_{r}}}_{x}$ $\overleftarrow{u_{2} L_{2} v_{1}}(o): \underbrace{b_{j_{1}} b_{j_{r}} b_{j_{r-1}} \overbrace{b_{j_{r-2}} \cdots b_{j_{3}}}^{x}}_{y} b_{j_{2}} \quad a_{j_{0}} b_{j_{0}} \quad a_{j_{1}} z \cdots \underbrace{a_{j_{r-3}} a_{j_{r-2}}}_{x} a_{j_{r-1}} a_{j_{r}}$

Case 16: $\left(s_{1}, s_{2}\right)=(2, r-1)$. In $v, z$ and $x$ are switched.

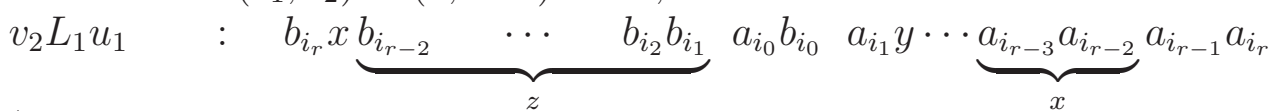

$\overleftarrow{u_{2} L_{2} v_{1}}(e): \quad b_{j_{r}} b_{j_{r-1}} \underbrace{b_{j_{r-2}} b_{j_{r-3}}}_{z} \cdots y b_{j_{1}} \quad a_{j_{0}} b_{j_{0}} \underbrace{a_{j_{1}} a_{j_{2}} \quad \cdots \quad a_{j_{r-2}}}_{x} z a_{j_{r}}$

To avoid the repetition $z y a_{j_{0}} \cdots z y a_{j_{0}}$ we choose new position for $z$ in $v$. $\overleftarrow{u_{2} L_{2} v_{1}}(o): \quad b_{j_{1}} b_{j_{r}} \underbrace{b_{j_{r-1}} b_{j_{r-2}}}_{z} \cdots b_{j_{3}} y a_{j_{0}} b_{j_{0}} \underbrace{a_{j_{1}} a_{j_{2}} \ldots \cdots a_{j_{r-2}}}_{x} z a_{j_{r}}$

Case 17: $\left(s_{1}, s_{2}\right)=(r-1,2)$ is symmetric to Case 16 .

Case 18: $\left(s_{1}, s_{2}\right)=(2, r)$. In $v, z$ and $y$ are switched.

$v_{2} L_{1} u_{1} \quad: \quad y b_{i_{r-1}} \underbrace{b_{i_{r-2}} \cdots b_{i_{2}}}_{x} b_{i_{1}} a_{i_{0}} b_{i_{0}} \quad a_{i_{1}} y a_{i_{3}} \cdots \underbrace{a_{i_{r-2}} a_{i_{r-1}} a_{i_{r}}}_{x} \quad\left[a_{i_{1}}\right]$

$\overleftarrow{u_{2} L_{2} v_{1}}(e):\left[b_{j_{1}}\right] \underbrace{b_{j_{r}} b_{j_{r-1}} b_{j_{r-2}}}_{x} \cdots b_{j_{3}} z b_{j_{1}} \quad a_{j_{0}} b_{j_{0}} \quad a_{j_{1}} \underbrace{a_{j_{2}} \cdots a_{j_{r-2}}}_{x} a_{j_{r-1}} z$

$\overleftarrow{u_{2} L_{2} v_{1}}(o): \quad b_{j_{1}} \underbrace{b_{j_{r}} b_{j_{r-1}} b_{j_{r-2}}}_{x} \cdots b_{j_{3}} z a_{j_{0}} b_{j_{0}} a_{j_{1}} \underbrace{a_{j_{2}} \quad \cdots \quad a_{j_{r-2}}}_{x} a_{j_{r-1}} z$

Case 19: $\left(s_{1}, s_{2}\right)=(r, 2)$. In $v, y$ and $x$ are switched.

$v_{2} L_{1} u_{1} \quad: \quad b_{i_{r}} \underbrace{b_{i_{r-1}} \cdots b_{i_{3}}}_{y} z b_{i_{1}} \quad a_{i_{0}} b_{i_{0}} \quad a_{i_{1}} \underbrace{a_{i_{2}} \cdots a_{i_{r-2}}}_{x} a_{i_{r-1}} y \quad\left[a_{i_{1}}\right]$

$\overleftarrow{u_{2} L_{2} v_{1}}(e):\left[b_{j_{1}}\right] x b_{j_{r-1}} \underbrace{b_{j_{r-2}} \cdots b_{j_{2}}}_{y} b_{j_{1}} a_{j_{0}} b_{j_{0}} a_{j_{1}} z \underbrace{a_{j_{3}} \cdots a_{j_{r-1}}}_{x} a_{j_{r}}$

$\overleftarrow{u_{2} L_{2} v_{1}}(o): \quad b_{j_{1}} x b_{j_{r-1}} \underbrace{b_{j_{r-2}} \cdots b_{j_{2}}}_{y} a_{j_{0}} b_{j_{0}} \quad a_{j_{1}} z \underbrace{a_{j_{3}} \cdots a_{j_{r-1}}}_{x} a_{j_{r}}$

Case 20: $\left(s_{1}, s_{2}\right) \in\{3, \ldots, r-2\} \times\{r-1\}$. In $v, z$ and $x$ are switched. $v_{2} L_{1} u_{1} \quad: \quad b_{i_{r}} x \underbrace{b_{i_{r-2}} \quad \cdots \quad b_{i_{1}}}_{z} a_{i_{0}} b_{i_{0}} \quad a_{i_{1}} \underbrace{a_{i_{2}} \overbrace{a_{i_{3}} \cdots a_{i_{r-2}}}^{y}}_{x} a_{i_{r-1}} a_{i_{r}}$ $\overleftarrow{u_{2} L_{2} v_{1}}(e): \quad b_{j_{r}} b_{j_{r-1}} \underbrace{\overbrace{b_{j_{r-2}} \cdots b_{j_{3}}}^{y} b_{j_{2}}}_{z} b_{j_{1}} a_{j_{0}} b_{j_{0}} \underbrace{a_{j_{1}} \quad \cdots \quad a_{j_{r-2}}}_{x} z a_{j_{r}}$ To avoid the repetition $y z a_{j_{0}} \cdots y z a_{j_{0}}$ we choose new position for $z$ in $v$. $\overleftarrow{u_{2} L_{2} v_{1}}(o): \quad b_{j_{1}} b_{j_{r}} \underbrace{b_{j_{r-1}} \overbrace{b_{j_{r-2}} \cdots b_{j_{3}}}^{y}}_{z} b_{j_{2}} a_{j_{0}} b_{j_{0}} \underbrace{a_{j_{1}} \ldots \ldots \quad a_{j_{r-2}}}_{x} z a_{j_{r}}$

Case 21: $\left(s_{1}, s_{2}\right) \in\{r-1\} \times\{3, \ldots, r-2\}$ is symmetric to Case 20.

Case 22: $\left(s_{1}, s_{2}\right) \in\{3, \ldots, r-2\} \times\{r\}$. In $v, y$ and $x$ are switched. 


$$
\begin{aligned}
& v_{2} L_{1} u_{1}(e): z b_{i_{r-1}} y \quad \cdots \quad b_{i_{1}} \quad a_{i_{0}} b_{i_{0}} \underbrace{a_{i_{1}} a_{i_{2}} \overbrace{a_{i_{3}} \cdots a_{i_{r-2}}} a_{i_{r-1}} a_{i_{r}}}_{x} \\
& \overleftarrow{u_{2} L_{2} v_{1}}(e): \underbrace{b_{j_{r}} b_{j_{r-1}} \overbrace{b_{j_{r-2}} \cdots b_{j_{3}}}^{x} b_{j_{2}} b_{j_{1}}}_{y} a_{j_{0}} b_{j_{0}} \quad a_{j_{1}} \quad \ldots \quad x a_{j_{r-1}} z
\end{aligned}
$$

To avoid the repetition $x y a_{j_{0}} \cdots x y a_{j_{0}}$ we choose new position for $y$ in $v$.

$$
\begin{aligned}
& v_{2} L_{1} u_{1}(o): \quad z b_{i_{r-1}} \underbrace{b_{i_{r-2}} b_{i_{r-3}}}_{y} \cdots b_{i_{1}} \quad a_{i_{0}} b_{i_{0}} \quad a_{i_{1}} \underbrace{a_{i_{2}} \overbrace{a_{i_{3}} \cdots a_{i_{r-2}}} a_{i_{r-1}} a_{i_{r}}}_{x} \\
& \overleftarrow{u_{2} L_{2} v_{1}}(o): \underbrace{b_{j_{1}} b_{j_{r}} b_{j_{r-1}} \overbrace{b_{j_{r-2}} \cdots b_{j_{3}}}^{x}}_{y} b_{j_{2}} \quad a_{j_{0}} b_{j_{0}} \quad a_{j_{1}} \quad \cdots \underbrace{a_{j_{r-3}} a_{j_{r-2}}}_{x} a_{j_{r-1}} z
\end{aligned}
$$

Case 23: $\left(s_{1}, s_{2}\right) \in\{r\} \times\{3, \ldots, r-2\}$ is symmetric to Case 22 .

Case 24: $\left(s_{1}, s_{2}\right)=(r-1, r)$. In $v, y$ and $x$ are switched.

$v_{2} L_{1} u_{1} \quad: \quad z b_{i_{r-1}} \underbrace{b_{i_{r-2}} \cdots b_{i_{2}}}_{y} b_{i_{1}} a_{i_{0}} b_{i_{0}} \quad a_{i_{1}} \underbrace{a_{i_{2}} \cdots a_{i_{r-2}}}_{x} y a_{i_{r}}$

$\overleftarrow{u_{2} L_{2} v_{1}}(e): \quad b_{j_{r}} x \underbrace{b_{j_{r-2} \cdots b_{j_{2}}}}_{y} b_{j_{1}} a_{j_{0}} b_{j_{0}} \quad a_{j_{1}} \underbrace{a_{j_{2}} \cdots a_{j_{r-2}}}_{x} a_{j_{r-1}} z$

$\overleftarrow{u_{2} L_{2} v_{1}}(o): \quad b_{j_{1}} b_{j_{r}} x \underbrace{b_{j_{r-2}} \cdots b_{j_{2}}}_{y} \quad a_{j_{0}} b_{j_{0}} \quad a_{j_{1}} \underbrace{a_{j_{2}} \cdots a_{j_{r-2}}}_{x} a_{j_{r-1}} z$

Case 25: $\left(s_{1}, s_{2}\right)=(r, r-1)$ is symmetric to Case 24 (if $n$ is odd, then we switch $x \rightarrow y \rightarrow z \rightarrow x$ in $v)$.

Now we verify that the constructed word $(w)$ is $((r+1) / r)^{+}$-free in all cases. By Observation 3, we exclude the factors $p t p$ such that the occurrences of $p$ are both in $u$ or both in $v$, from our consideration. Let us check the remaining factors. By construction, each of the words $u_{1}, u_{2}, v_{1}$, and $v_{2}$ contains exactly two of the letters $x, y$, and $z$. In all cases except Case 18, it can be assumed w.l.o.g. that $x$ appears in both $v_{2}$ and $u_{1}, y$ is not in $v_{2}$, and $z$ is not in $u_{1}$ (the pair $\left(u_{2}, v_{1}\right)$ is studied in the same way). Below all repetitions with their exponents are listed (repetitions that have different exponents in Case 18 are given in brackets).

$$
\begin{aligned}
& x \underbrace{\cdots}_{\geqslant r-1} x \\
& y \underbrace{\cdots v_{2} L_{1} \cdots}_{\geqslant r+2} y[y \underbrace{\cdots}_{\geqslant r+1} y] \\
& \Rightarrow \quad \exp (p t p) \leqslant(r+1) / r \\
& z \underbrace{\cdots L_{1} u_{1} \cdots}_{\geqslant r+2} z[z \underbrace{\cdots v_{2} L_{1} u_{1} \cdots}_{\geqslant 2 r+2} z] \\
& z x \underbrace{\cdots}_{\geqslant r-1} x \underbrace{\cdots}_{\geqslant r-2} z x[z x \underbrace{\cdots}_{\geqslant r-1} x \underbrace{\cdots}_{\geqslant r-1} x \underbrace{\cdots}_{\geqslant r-2} z x] \Rightarrow \exp (p t p) \leqslant(2 r+2) /(2 r) ; \\
& y x \underbrace{\cdots}_{\geqslant r-1} x \underbrace{\cdots}_{\geqslant r-2} y x[y x \underbrace{\cdots}_{\geqslant r-1} x \underbrace{\cdots}_{\geqslant r-1} x \underbrace{\cdots}_{\geqslant r-2} y x] \Rightarrow \exp (p t p) \leqslant(2 r+2) /(2 r) \text {; }
\end{aligned}
$$


$y z \underbrace{\cdots v_{2}^{\prime} L_{1} u_{1}^{\prime} \cdots}_{\geqslant 2 r} y z[z y \underbrace{\cdots}_{\geqslant r+1} y \underbrace{\cdots}_{\geqslant r-2} z y] \Rightarrow \exp (p t p) \leqslant(2 r+4) /(2 r+2)$,

here $u_{1}^{\prime}$ equals either $u_{1}$ or $u_{1}$ without the letter $a_{i_{r}}$; the same for $v_{2}$;

$x y z \underbrace{\cdots}_{\geqslant r-3} x \underbrace{\cdots}_{\geqslant r-1} x \underbrace{\cdots}_{\geqslant r-1} x y z \quad \Rightarrow \quad \exp (p t p) \leqslant(3 r+3) /(3 r)$

(in Case 12(o), we rely on $z$ 's in the middle instead of $x$ 's).

Suppose $z$ appears in the first position; then

$z a_{i_{0}} \underbrace{\cdots}_{\geqslant r-1} a_{i_{r-1}}\left(a_{i_{r}}\right) \underbrace{\ldots}_{\geqslant r-2} z a_{i_{0}} \quad \Rightarrow \exp (p t p) \leqslant(2 r+2) /(2 r)$.

The following repetitions are always allowed by the choice of $a_{i_{0}}$ (similarly for $a_{j_{0}}, b_{i_{0}}, b_{j_{0}}$ ):

$\begin{array}{ll}a_{i_{0}} \underbrace{\cdots}_{\underbrace{\cdots}_{r-1}} a_{i_{r-1}}\left(a_{i_{r}}\right) & \Rightarrow \exp \leqslant(r+1) / r ; \\ a_{i_{0}} b_{i_{0}} \underbrace{\cdots}_{\geqslant r-2} a_{i_{r-1}}\left(a_{i_{r}}\right) \underbrace{\cdots}_{\geqslant r-1} a_{j_{1}} b_{j_{0}} & \Rightarrow \exp \leqslant(2 r+2) / 2 r .\end{array}$

Recall that $r=\lceil k / 2\rceil \geqslant 6$. If in Case I there is a repetition $b_{j_{0}} a_{j_{0}} b_{j_{1}} \cdots b_{i_{1}} a_{i_{0}} b_{i_{0}}$ [or symmetrically $\left.a_{i_{0}} b_{i_{0}} a_{i_{1}} \cdots a_{j_{1}} b_{j_{0}} a_{j_{0}}\right]$, then $a_{j_{r}}=a_{j_{0}}=a_{i_{0}} \in\left\{a_{i_{r-1}}, a_{i_{r}}\right\}$ [resp., $b_{i_{r}} \in\left\{b_{j_{r-1}}, b_{j_{r}}\right\}$ if $n$ is even, $b_{i_{r}} \in\left\{b_{j_{1}}, b_{j_{r}}\right\}$ if $n$ is odd]. Hence, there are at least $r-1$ letters between $a_{i_{r-1}}$ and $a_{j_{r}}$. So we have $u_{1} \underbrace{a_{i_{1}} z \cdots \overbrace{a_{j_{r+2}} a_{j_{r+1}}}^{z}}_{\geqslant r-2} u_{2}$. There are at least $r-1$ letters between the two occurrences of $z$, whence $|v| \geqslant|u|-1 \geqslant 3 r+1$ [resp., $|u| \geqslant|v| \geqslant 3 r+1$ ] and $b_{j_{0}} a_{j_{0}} b_{j_{1}} \underbrace{\cdots}_{\geqslant|v|-2} b_{i_{1}} a_{i_{0}} b_{i_{0}}[$ resp., $a_{i_{0}} b_{i_{0}} a_{i_{1}} \underbrace{\cdots}_{\geqslant|u|-2} a_{j_{1}} b_{j_{0}} a_{j_{0}}]$. Thus, $\exp (p t p) \leqslant(3 r+5) /(3 r+2)<$ $(r+1) / r$. Similarly, we obtain $u_{1} \underbrace{y \cdot \cdots \overbrace{a_{j_{r+2}} a_{j_{r+1}}}^{y}}_{\geqslant r-1} u_{2}$ in Case $6(\mathrm{o}), a_{i_{1}} \cdots y \underbrace{\cdots \overbrace{a_{j_{r+2}} a_{j_{r+1}}}^{y}}_{\geqslant r-3} u_{2}$ in Cases 19 and 23, and $u_{1} \underbrace{a_{i_{1}} z \cdots}_{\geqslant r-3} \overbrace{a_{j_{r}} a_{j_{r-1}}}^{z} \cdots a_{j_{1}}$ in Cases 4, 5, 10(o), 12(o), 18, 22, 24, and 25. Hence, in all these cases $|v| \geqslant|u|-1 \geqslant 3 r-1$ and $b_{j_{0}} a_{j_{0}} b_{j_{1}} \underbrace{\cdots}_{\geqslant|v|-2} b_{i_{1}} a_{i_{0}} b_{i_{0}}$ [resp., $|u| \geqslant|v| \geqslant 3 r-1$ and $a_{i_{0}} b_{i_{0}} a_{i_{1}} \underbrace{\ldots}_{\geqslant|u|-2} a_{j_{1}} b_{j_{0}} a_{j_{0}}]$. Thus, $\exp (p t p) \leqslant(3 r+3) /(3 r)=(r+1) / r$. So, we proved the existence of $((r+1) / r)^{+}$-free circular words of any length $n \geqslant 2 k+6$.

In order to complete the proof of the theorem, it remains to consider the case $k+1 \leqslant n \leqslant 2 k+5$. If $2 k+2 \leqslant n \leqslant 2 k+5$, then we construct $w=L_{1} u L_{2} v$, where $u \in A^{*}$ and $v \in B^{*}$ are arbitrary $((r+1) / r)^{+}$-free words such that $|u|=\lceil(n-4) / 2\rceil, u[1]=$ $u[r+1],|v|=\lfloor(n-4) / 2\rfloor$ and $v[1]=v[r+1]$. Thus we know that $u[r+2]=\Sigma\left(u_{2}\right) \backslash \Sigma\left(u_{1}\right)$ and $\{\overleftarrow{u}[r+1], \overleftarrow{u}[r+2]\} \cap\left(\Sigma\left(u_{1}\right) \backslash \Sigma\left(u_{2}\right)\right) \neq \varnothing$. So for $n=2 k+5$ and $n=2 k+3$ we switch letters in $u$ and $v$ as shown in Fig. 2. If $n=2 k+4$, we take $L_{1}=a_{i_{r}} b_{j_{r}}, L_{2}=b_{j_{r}} a_{i_{r}}, u$ from the case $n=2 k+3$, and $v$ from the case $n=2 k+5$. If $n=2 k+2$, we take $L_{1}=a_{i_{r-1}} b_{j_{r-1}}$, $L_{2}=b_{j_{r-1}} a_{i_{r-1}}$, and $u=f^{-1}(v)$, where $v$ is from the case $n=2 k+3$.

If $n \leqslant 2 k+1$, we abandon the "buffers" $L_{1}, L_{2}$ and put $w=u v$ (see the bottom row of pictures in Fig. 2). If $n=2 k+1$, then we take $u$ and $v$ from the case $n=2 k+5$, and 

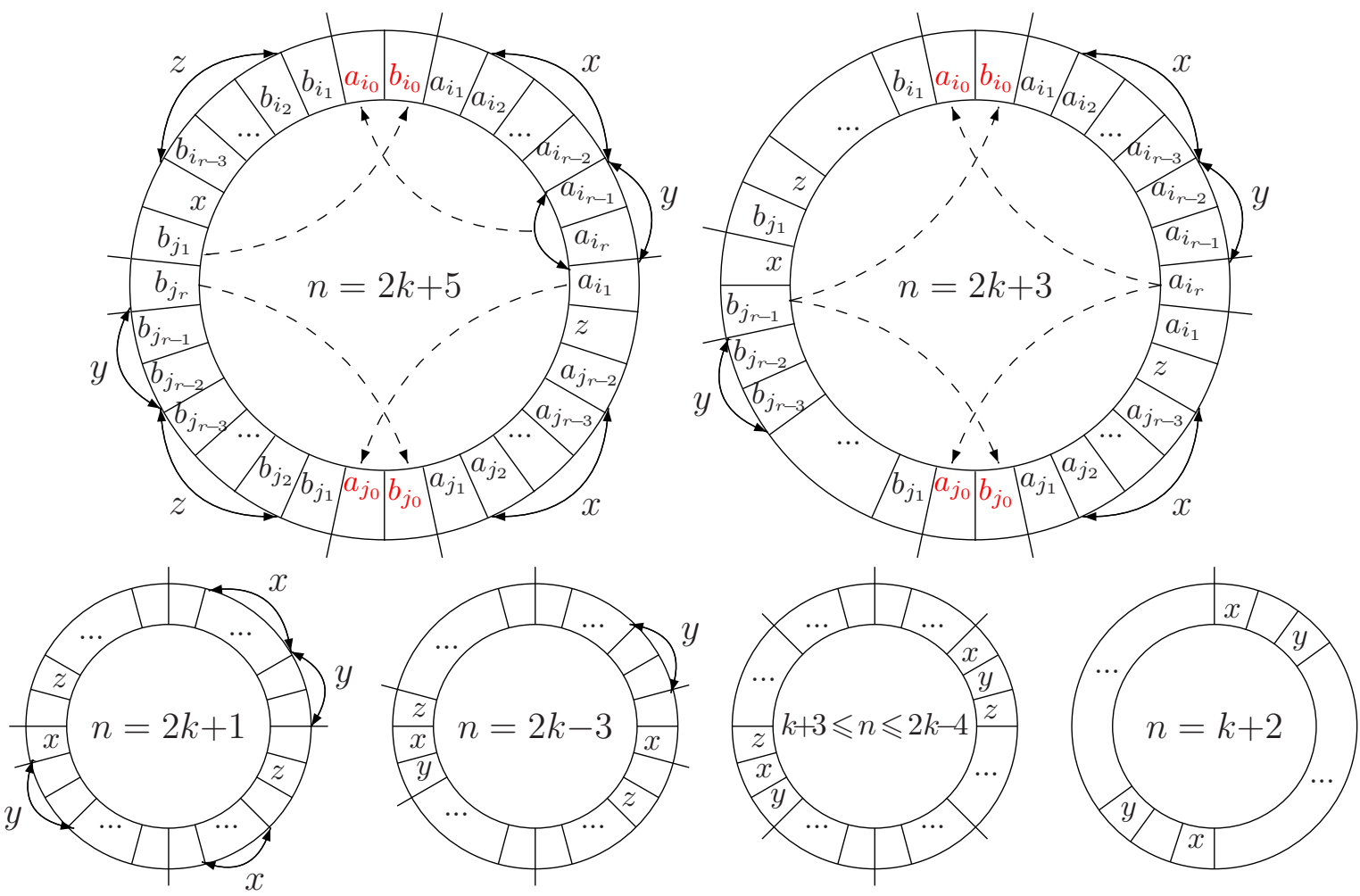

Figure 2: Circular word $(w)$ of length $n(k+1 \leqslant n \leqslant 2 k+5)$.

switch $z$ and $x ; x$ and $b_{j_{r}}$ in $v$. If $n=2 k$, then we take $u=f^{-1}(v)$, where $v$ is from the case $n=2 k+1$, and switch $z$ and $y$ in $u$. If $n=2 k-1$, then we take $u$ from the case $n=2 k$ and $v$ from the case $n=2 k+3$. If $n=2 k-2$, then we take $u=f^{-1}(v)$, where $v$ is from the case $n=2 k-1$. For $k+3 \leqslant n \leqslant 2 k-3$, we switch the letters in $u$ and $v$ as shown in Fig. 2. If $n=k+2$, then we construct $(w)$ such that $w[1]=w[r+1]=x$, $w[3]=w[r+3]=y$, and any other letter occurs in $w$ only once. If $n=k+1$, then we construct $(w)$ such that $w[1]=w[r+1]=x$ and any other letter occurs in $w$ only once. It is easy to check that the constructed word $(w)$ is $((r+1) / r)^{+}$-free in all cases.

\section{Special cases}

The cases $k=6, k=7$, and $k=9$ are studied separately because the proof of Theorem 2 uses the inequality $\lceil k / 2\rceil \geqslant 6$. We follow the main line of that proof but must consider more cases.

Theorem 4. There exist circular $(4 / 3)^{+}$-free 6 -ary words of any length.

Proof. The $(4 / 3)^{+}$-free circular words of length $7, \ldots, 19$ are shown in Fig. 3. For $n \geqslant 20$ we construct such a word $(w)$ in the form $w=16 u 61 v$, where $u \in\{1, \ldots, 5\}^{*}$ and $v \in\{2, \ldots, 6\}^{*}$ are $(5 / 4)^{+}$-free words bound by the same conditions as in the proof of Theorem 2 (the bijection $f$ such that $v=f(u)$ maps 1 to 6 and fixes other letters). Let $u_{1}$ 
and $u_{2}$ be the prefix and the suffix of $u$ of the length 4 . There are 120 ways to place equal letters into $u_{1}$ and $u_{2}$ (see Fig. 4). For each of these variants we construct the word $(w)$.
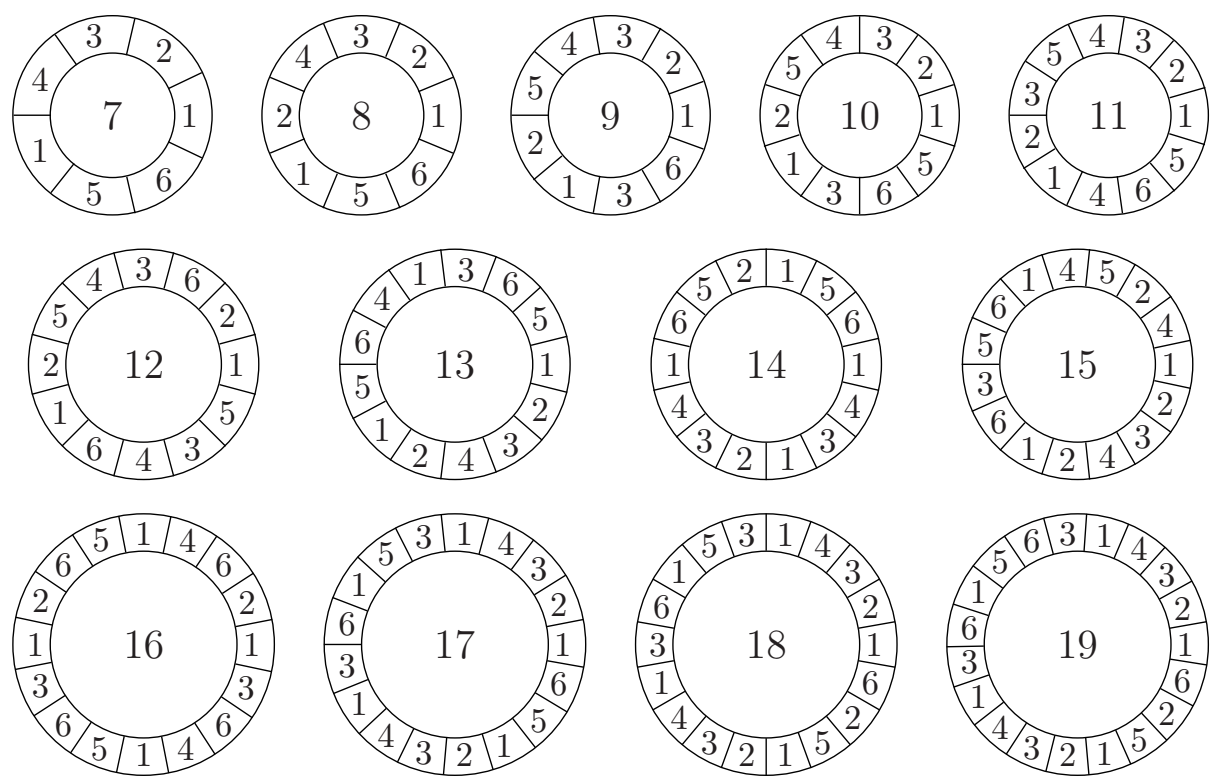

Figure 3: Circular $(4 / 3)^{+}$-free words of length $n(7 \leqslant n \leqslant 19)$ over 6 letters.

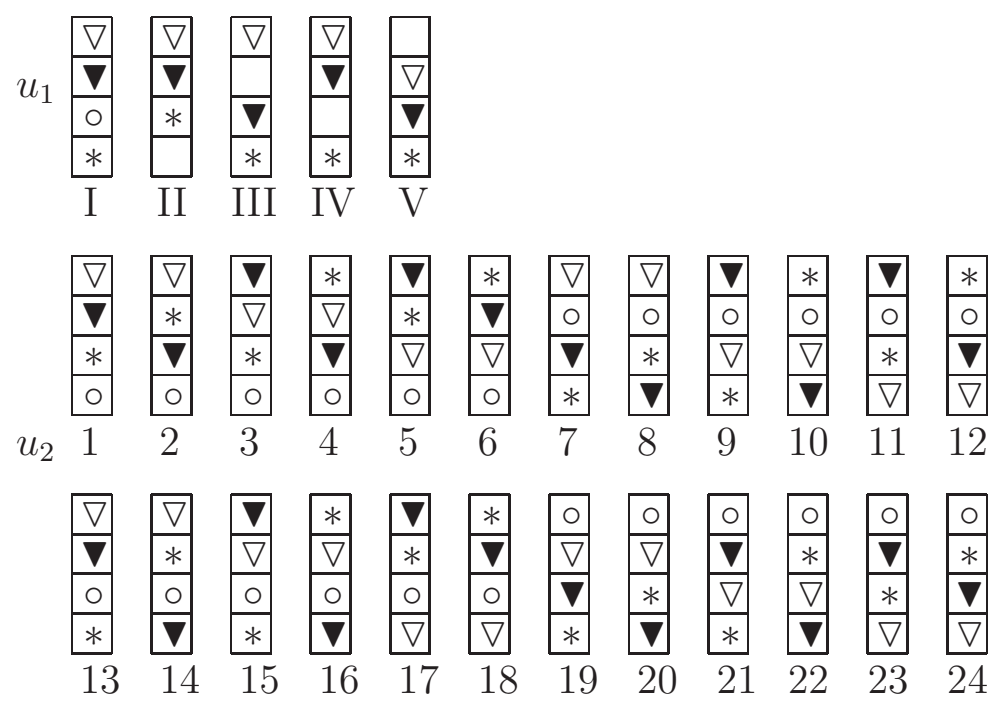

Figure 4: Ways of placing equal letters into $u_{1}$ and $u_{2}$ for $k=6$.

By Observation 3, it is sufficient to prove that any factor of $w$ of the form $p t p$ such that both occurrences of $p$ are not inside the same word ( $u$ or $v)$, has allowed exponent. Since $|A \cap B|=4$, we see that the length of the longest common factor of $u$ and $v$ is 5 (this is the factor 23452, up to renaming the letters). Due to the "buffers" 16 and 61, we need to check only the repetitions with $|p| \leqslant 6$ ( $p=6$ only in the case when $p$ contains a letter from the buffer). To avoid these forbidden repetitions, we permute the letters in $u$ 


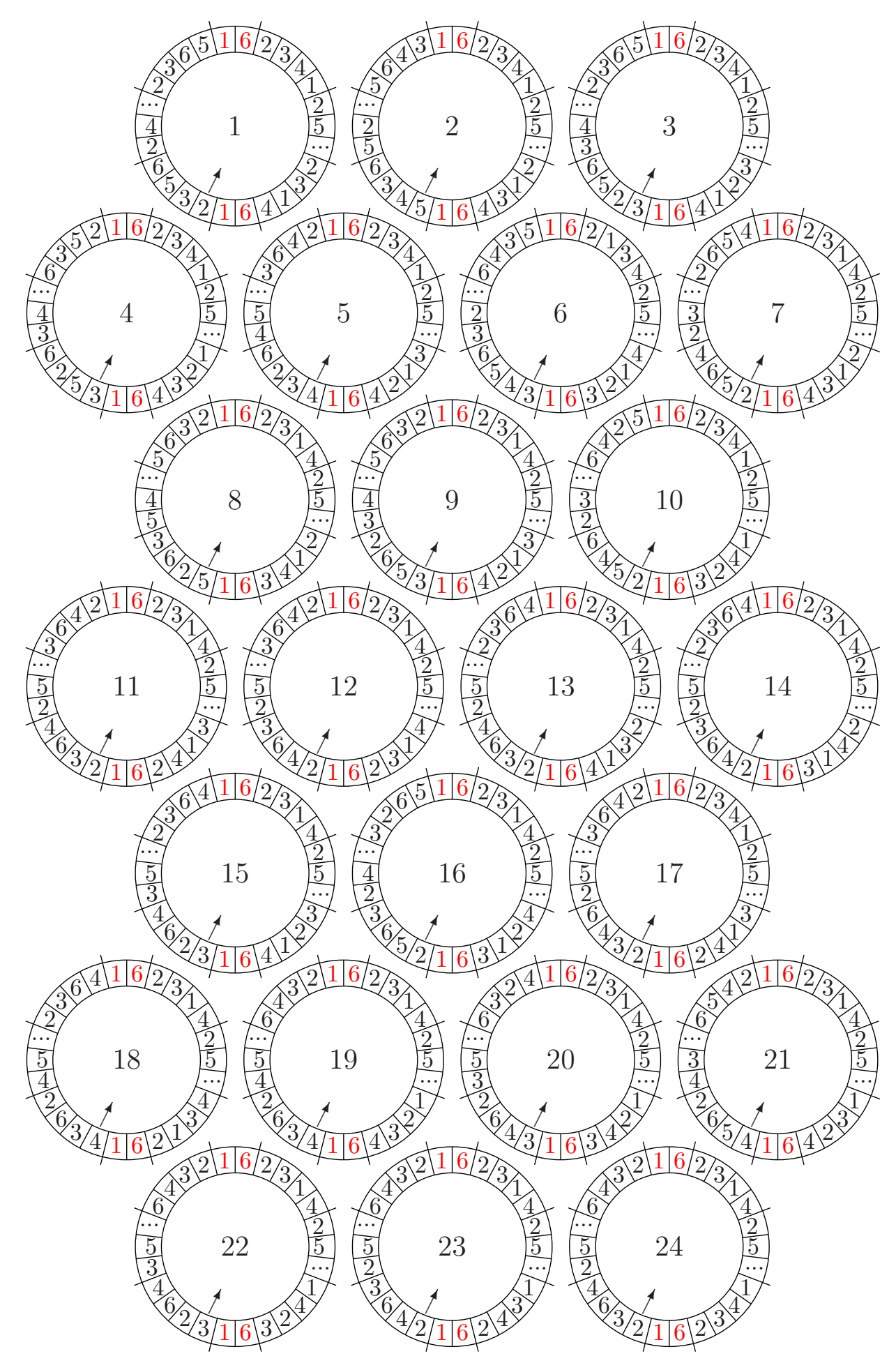

Figure 5: Circular $(4 / 3)^{+}$-free words of length $n(n \geqslant 20)$ over 6 letters. The word $u_{1}$ is of type I. The type of $u_{2}$ is written in the center of the circle. These diagrams describe the case when $n$ is even. If $n$ is odd, then the word of length $n$ is obtained from the word of length $n+1$ by removing the first letter of $v$ (marked with an arrow). 


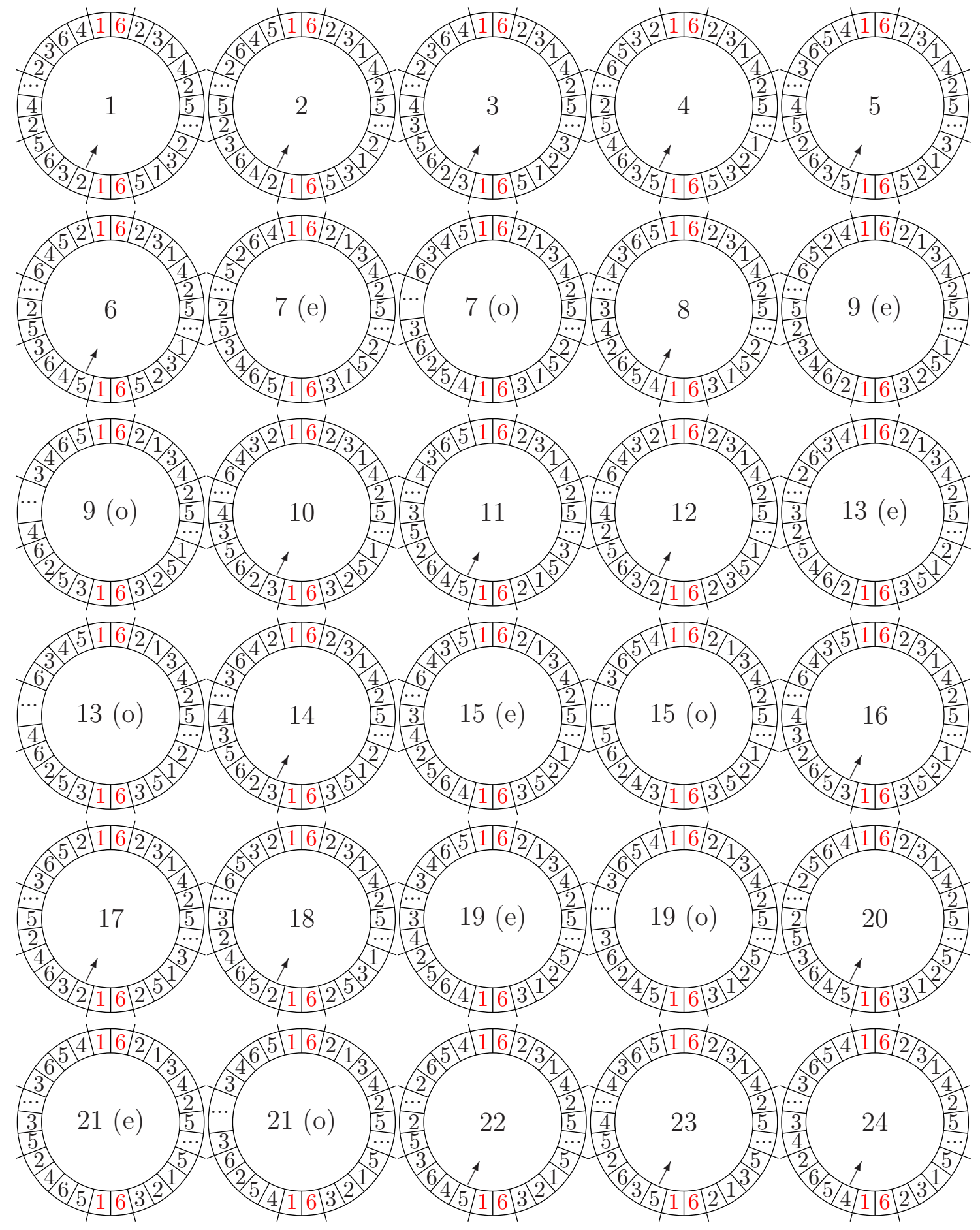

Figure 6: Circular $(4 / 3)^{+}$-free words of length $n(n \geqslant 20)$ over 6 letters. The word $u_{1}$ is of type II. The type of $u_{2}$ is written in the center of the circle. In some cases, these words are constructed separately for even and odd $n$. 

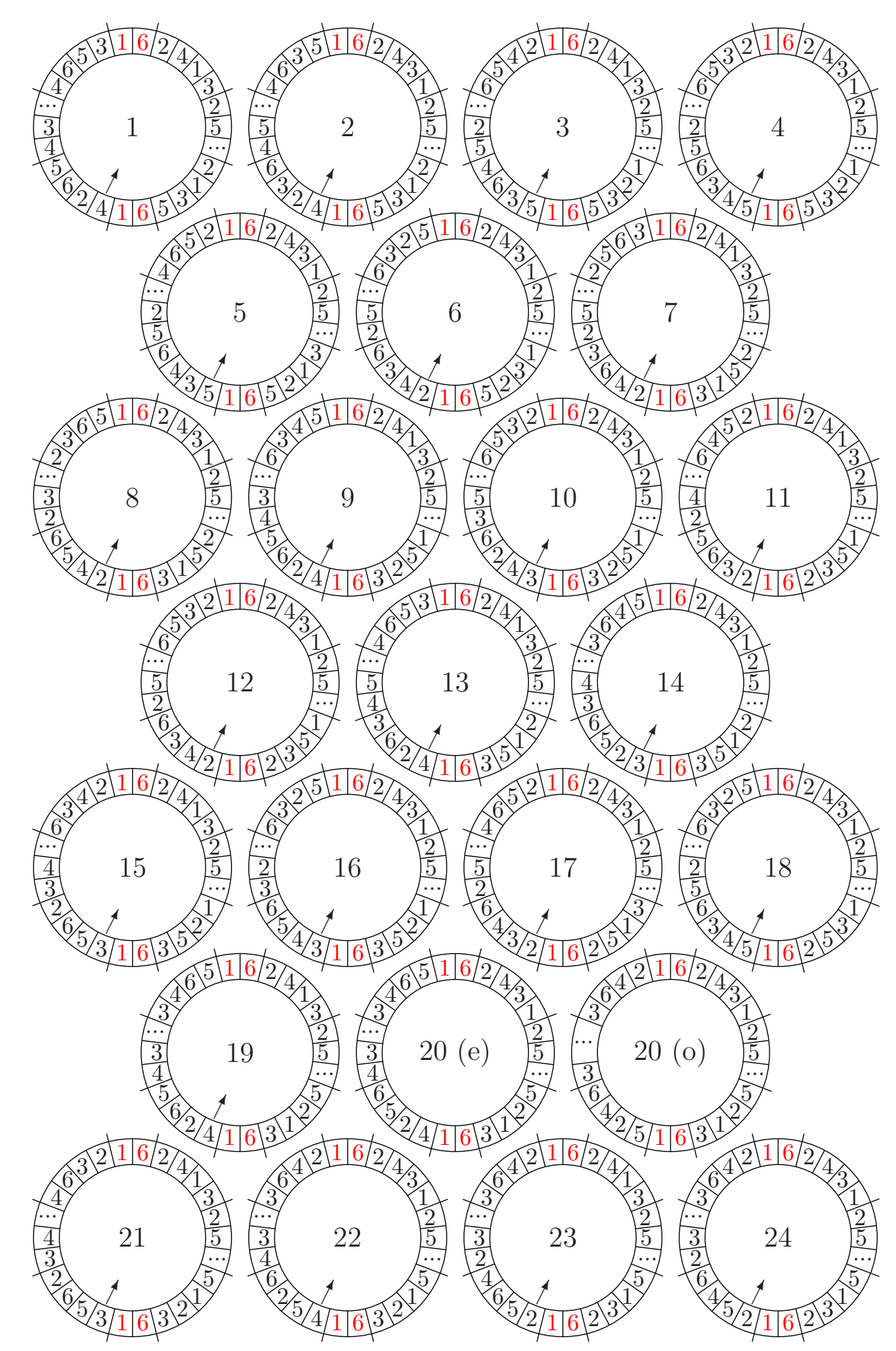

Figure 7: Circular $(4 / 3)^{+}$-free words of length $n(n \geqslant 20)$ over 6 letters. The word $u_{1}$ is of type III. The type of $u_{2}$ is written in the center of the circle. 

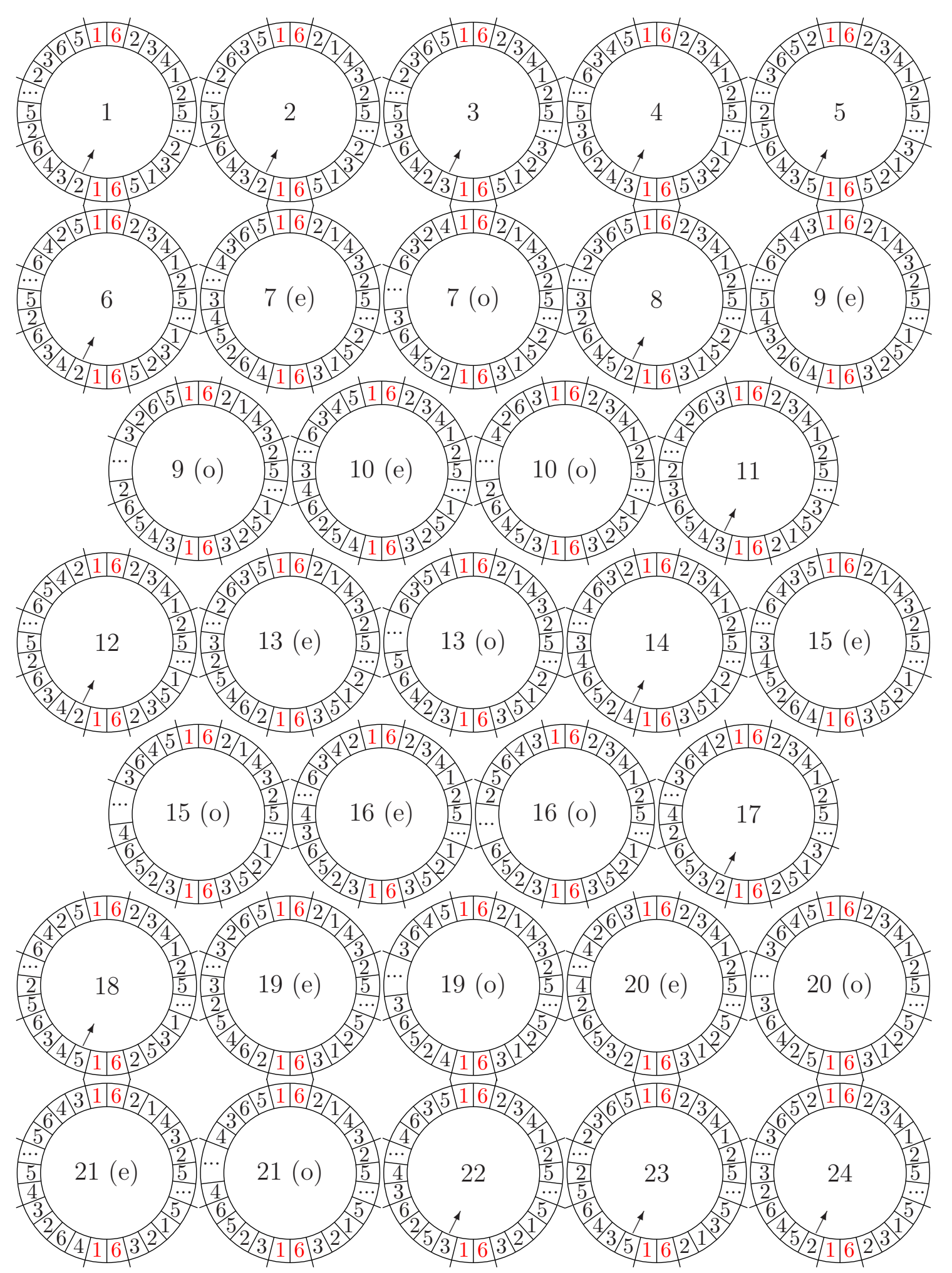

Figure 8: Circular $(4 / 3)^{+}$-free words of length $n(n \geqslant 20)$ over 6 letters. The word $u_{1}$ is of type IV. The type of $u_{2}$ is written in the center of the circle. Note that in Case 16(o) we take $\overleftarrow{v}$ instead of $v$ 


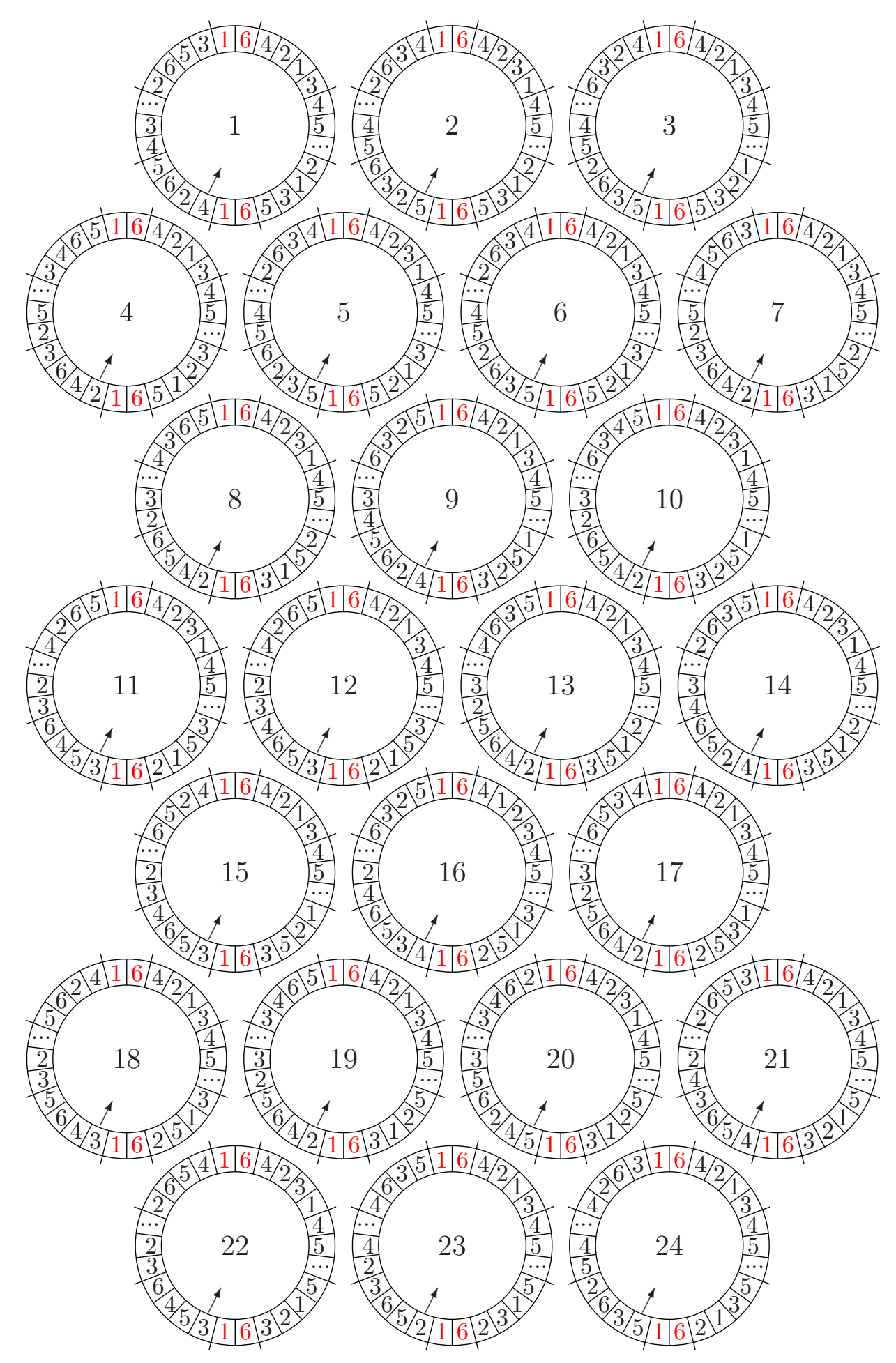

Figure 9: Circular $(4 / 3)^{+}$-free words of length $n(n \geqslant 20)$ over 6 letters. The word $u_{1}$ is of type $V$. The type of $u_{2}$ is written in the center of the circle. 
and $v$ such that their prefixes and suffixes look like in Fig. 5-9. In any of the 120 cases, the depicted prefix and suffix of $u$ [resp., $v$ ] contains 1 [resp., 6]. Such structure of $u$ and $v$ guarantees that $p \leqslant 5$ and $p=5$ only if one occurrence of $p$ is inside $u$ and the other one is inside $v$. The remaining candidates for the factor of exponent greater than $4 / 3$ can be easily checked in each case.

In order to reduce the number of possible cases for $k=7$ and $k=9$, we increase the length of $L_{1}$ and $L_{2}$. Such an increment allows one to make the words $u_{1}, u_{2}, v_{1}$, and $v_{2}$ shorter and to omit the requirement $u[1]=u[\lceil k / 2\rceil+1])$.

Theorem 5. There exist circular $(5 / 4)^{+}$-free 7 -ary words of any length.

Proof. The $(5 / 4)^{+}$-free circular words of length $8, \ldots, 17$ are shown in Fig. 10 . For $n \geqslant 18$ we construct such a word $(w)$ in the form $w=L_{1} u L_{2} v$, where $L_{1}, L_{2} \in\{1,2,6,7\}^{4}$, $u \in\{1, \ldots, 5\}^{*}$ and $v \in\{3, \ldots, 7\}^{*}$ are $(5 / 4)^{+}$-free words, $v=f(u)$ for a bijection $f:\{1, \ldots, 5\} \rightarrow\{3, \ldots, 7\}$ (for odd $n, v$ equals $f(u)$ without the first letter). Let $u_{1}, u_{2}$, $v_{1}$, and $v_{2}$ be the prefixes of length 3 and the suffixes of length 2 of $u$ and $v$, respectively. There are 13 ways to place equal letters into $u_{1}$ and $u_{2}$ (see Fig. 11). We call these ways the types of $u$. If $n$ is even, then the type of $v$ coincides with the type of $u$; if $n$ is odd, then the type of $v$ depends on the type of $u$ according to Table 1.
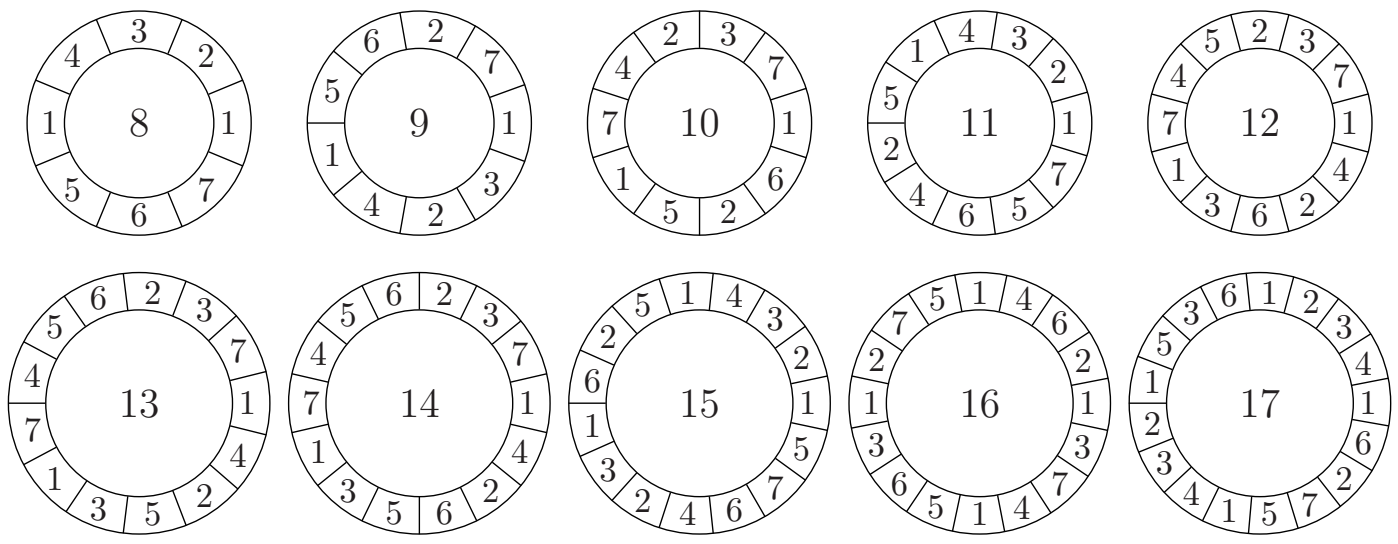

Figure 10: Circular $(5 / 4)^{+}$-free words of length $n(8 \leqslant n \leqslant 17)$ over 7 letters.

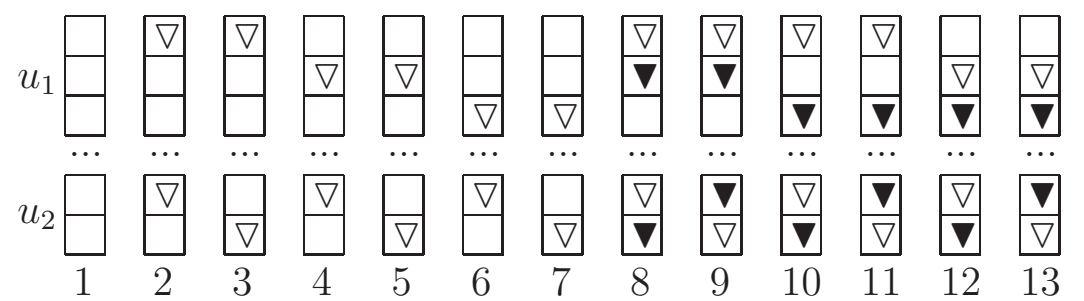

Figure 11: Ways of placing equal letters into $u_{1}$ and $u_{2}$ for $k=7$ (Type of $u$ ).

By Observation 3, it is sufficient to prove that any factor of $w$ of the form $p t p$ such that both occurrences of $p$ are not inside the same word ( $u$ or $v$ ), has allowed exponent. 


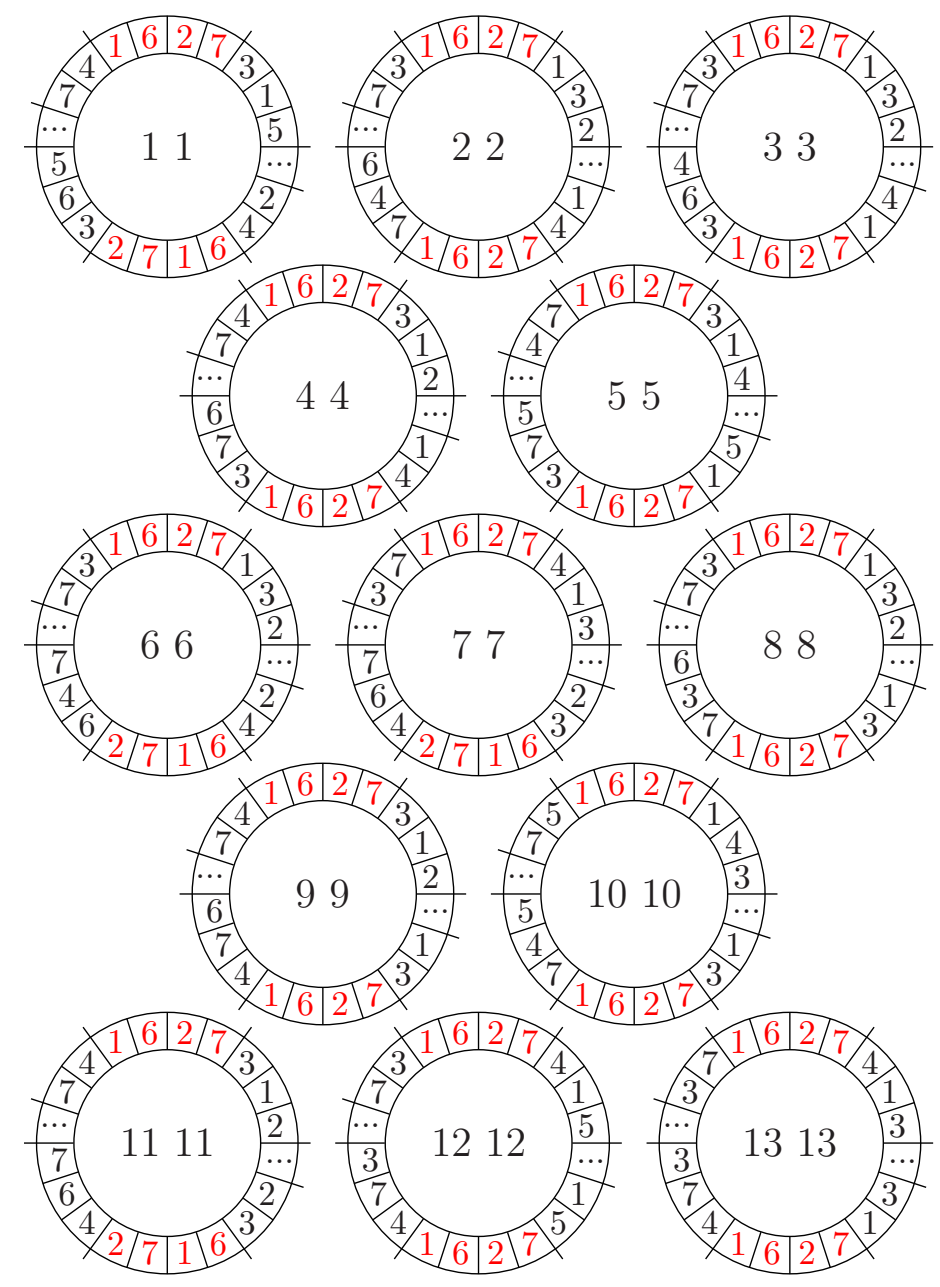

Figure 12: Circular (5/4)+-free words of length $n$ ( $n \geqslant 18$ is even) over 7 letters. The types of $u$ and $v$ are given in the center of the circle.

Table 1: Transition table for $k=7$. Types of $u$ and the corresponding types of $v$ in the case of odd $n$ are given.

\begin{tabular}{|c|c|c|c|c|c|c|c|c|c|c|c|c|c|}
\hline Type of $u$ & 1 & 2 & 3 & 4 & 5 & 6 & 7 & 8 & 9 & 10 & 11 & 12 & 13 \\
\hline Possible & 6 & 1 & 1 & 2 & 3 & 4 & 5 & 3 & 2 & 5 & 4 & 8 & 9 \\
types of $v$ & 7 & 7 & 6 & 10 & 11 & 12 & 13 & & & & & & \\
\hline
\end{tabular}




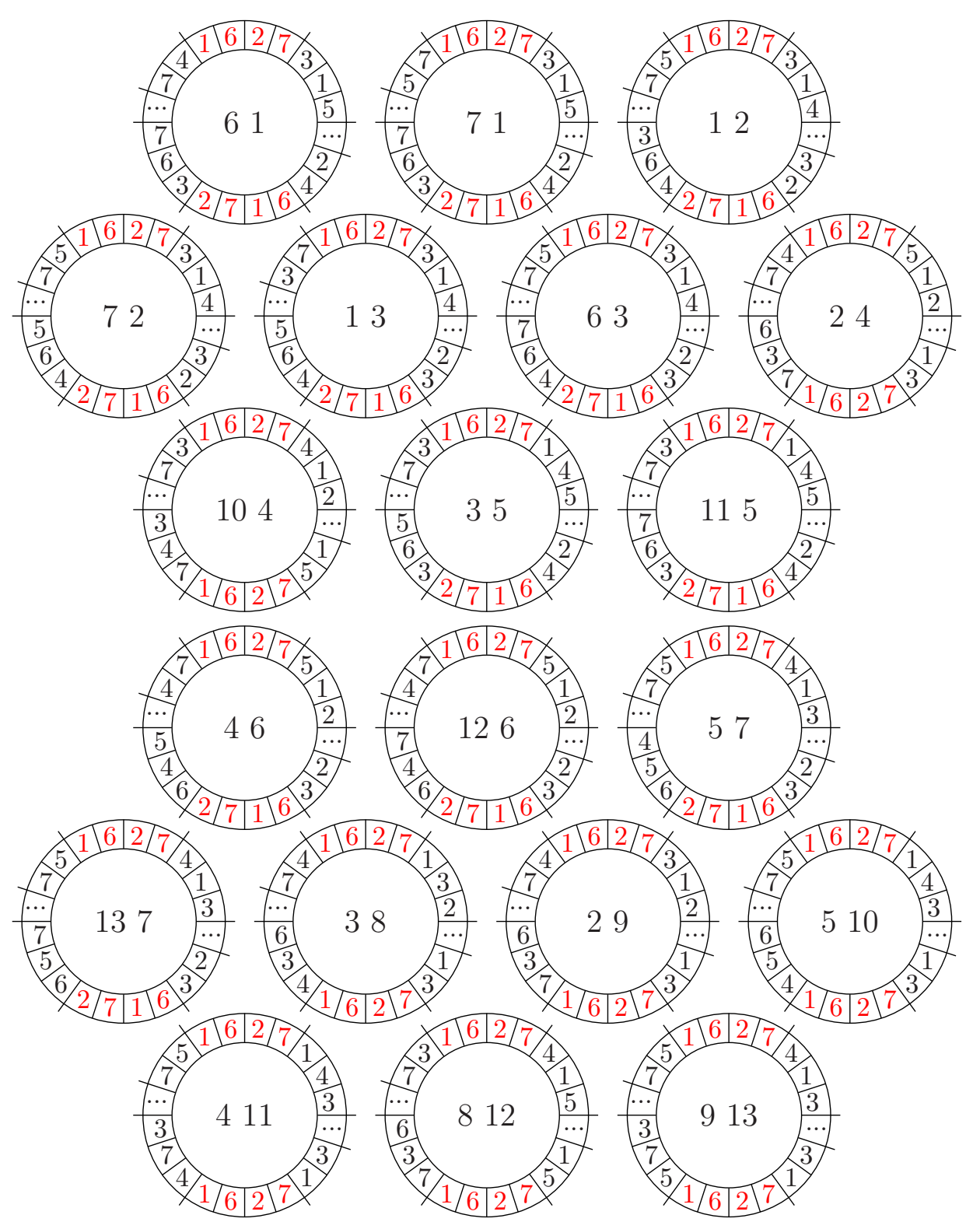

Figure 13: Circular (5/4)+-free words of length $n(n \geqslant 18$ is odd) over 7 letters. In some cases, the proposed construction does not allow to avoid all forbidden factors. The following forbidden repetitions can appear in the word $(w): 4537162731453$ (Case (1 3)), 35471627512354 (Cases (4 6) and (12 6)), $34162731 \underbrace{\ldots}_{2-3} 341$ (Case (3 8)), $54162731 \underbrace{\ldots}_{1-3} 541$ (Case (5 10)). If such repetitions appear in $(w)$, then in order to avoid them, we make an additional switch in $u$ only. For Case (5 10), we switch 2 and 5; for all other cases, we switch 4 and 5. 
Since $|A \cap B|=3$, we see that the length of the longest common factor of $u$ and $v$ is 3 (this is the factor 345, up to renaming the letters). Due to the "buffers" $L_{1}$ and $L_{2}$, we need to check only the repetitions with $|p| \leqslant 4$ ( $p=4$ only in the case when $p$ contains a letter from the buffer). To avoid these forbidden repetitions, we permute the letters in $u$ and $v$ such that their prefixes and suffixes look like in Fig. 12 and 13. The remaining candidates for the factor of exponent greater than $5 / 4$ can be easily checked in each case.

Theorem 6. There exist circular $(6 / 5)^{+}$-free 9 -ary words of any length.

Proof. The $(6 / 5)^{+}$-free circular words of length 10, ., 23 are shown in Fig. 14. For $n \geqslant 24$ we construct such a word $(w)$ in the form $w=L_{1} u L_{2} v$, where $L_{1}, L_{2} \in\{1,2,3,7,8,9\}^{6}$, $u \in\{1, \ldots, 6\}^{*}$ and $v \in\{4, \ldots, 9\}^{*}$ are $(6 / 5)^{+}$-free words, $v=f(u)$ for a bijection $f:\{1, \ldots, 6\} \rightarrow\{4, \ldots, 9\}$ (for odd $n, v$ equals $f(u)$ without the first letter). Let $u_{1}, u_{2}$, $v_{1}$, and $v_{2}$ be the prefixes and the suffixes of length 3 of $u$ and $v$, respectively. There are 34 ways to place equal letters into $u_{1}$ and $u_{2}$ (see Fig. 15). If $n$ is even, then the type of $v$ coincides with the type of $u$; if $n$ is odd, then the type of $v$ depends on the type of $u$ according to Table 2 .

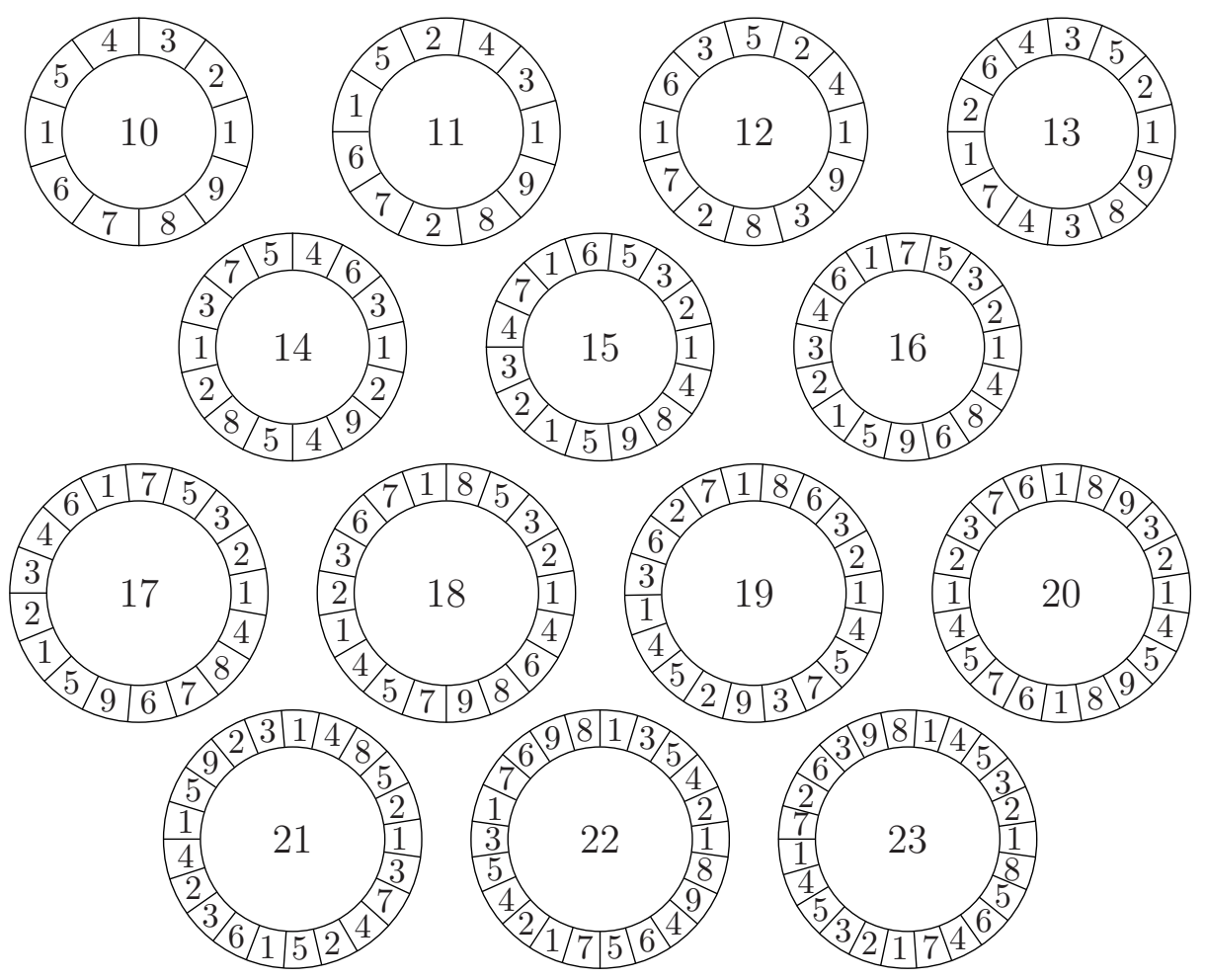

Figure 14: Circular $(6 / 5)^{+}$-free words of length $n(10 \leqslant n \leqslant 23)$ over 9 letters.

By Observation 3, it is sufficient to prove that any factor of $w$ of the form $p t p$ such that both occurrences of $p$ are not inside the same word ( $u$ or $v)$, has allowed exponent. Since $|A \cap B|=3$, we see that the length of the longest common factor of $u$ and $v$ is 3 (this is the factor 456, up to renaming the letters). Due to the "buffers" $L_{1}$ and $L_{2}$, we 
need to check only the repetitions with $|p| \leqslant 4$ ( $p=4$ only in the case when $p$ contains a letter from the buffer). To avoid these forbidden repetitions, we permute the letters in $u$ and $v$ such that their prefixes and suffixes look like in Fig. 16-18.

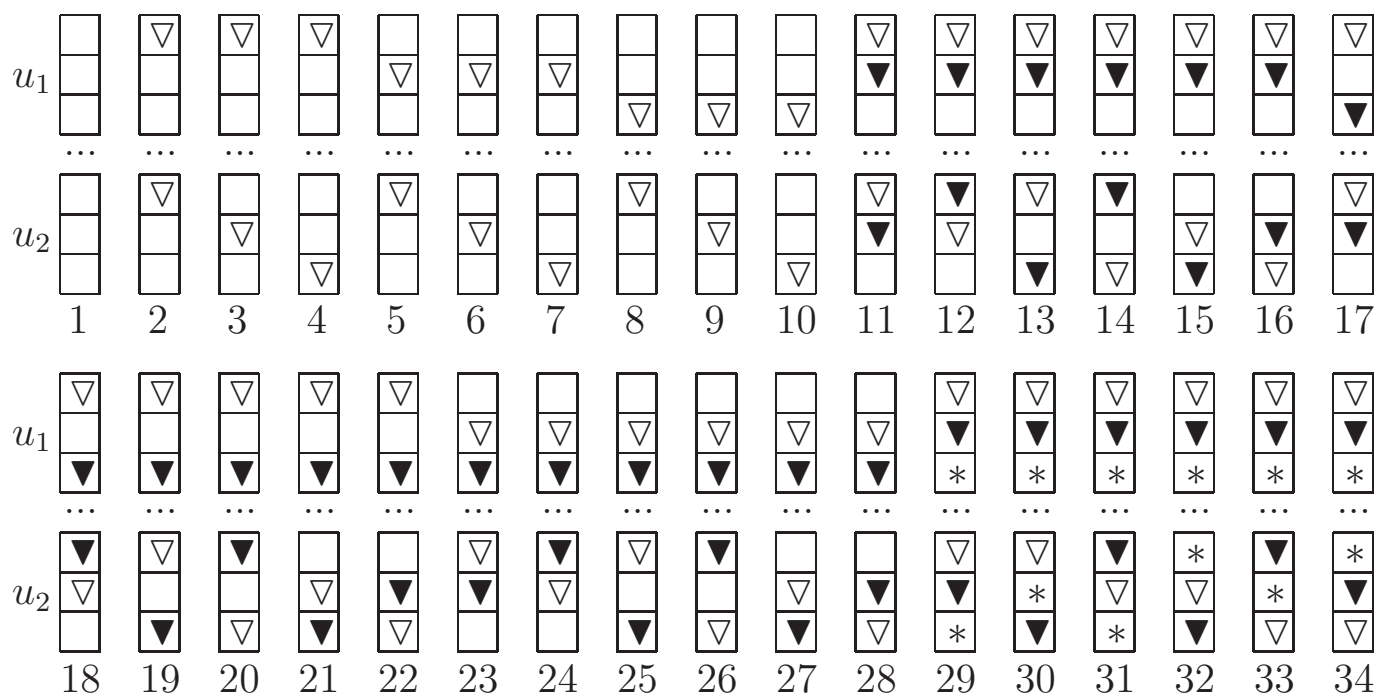

Figure 15: Ways of placing equal letters into $u_{1}$ and $u_{2}$ for $k=9$ (Type of $u$ ). Types 2 and 10; 3 and 7; 5 and 9; 11 and 27; 12 and 28; 13 and 21; 14 and 22; 17 and 25; 18 and 26; 30 and 31 coincide up to reversal. So we need to consider only 24 types.

Table 2: Transition table for $k=9$. Types of $u$ and the corresponding types of $v$ in the case of odd $n$ are given.

\begin{tabular}{|c|c|c|c|c|c|c|c|c|c|c|c|c|c|c|c|c|c|c|c|c|c|c|c|c|}
\hline Type of $u$ & 1 & 2 & 3 & 4 & 5 & 6 & 8 & 11 & 12 & 13 & 14 & 15 & 16 & 17 & 18 & 19 & 20 & 23 & 24 & 29 & 30 & 32 & 33 & 34 \\
\hline \multirow{3}{*}{ Possible types of $v$} & 2 & 1 & 1 & 1 & 2 & 3 & 5 & 3 & 2 & 4 & 2 & 4 & 3 & 6 & 5 & 3 & 5 & 11 & 12 & 15 & 16 & 14 & 11 & 12 \\
& 5 & 2 & 5 & 17 & 13 & 17 & 13 & 19 & 14 & 17 & 20 & 18 & 11 & 17 & 12 & 23 & 29 & 30 & & & & & & \\
& 8 & 5 & 8 & 8 & 19 & 18 & 23 & & & & & & & & & & & & & & & & & \\
\hline
\end{tabular}

Finally, we put all the obtained results together.

Corollary 7. For any $k \geqslant 6, \operatorname{CRT}(k)=\frac{\lceil k / 2\rceil+1}{\lceil k / 2\rceil}$.

Proof. Combining Proposition 1 with Theorems 2,4,5,6, we get the required formula for $k=6$ and for any odd $k \geqslant 7$. If $k$ is even, then $\frac{\lceil k / 2\rceil+1}{\lceil k / 2\rceil}=\frac{\lceil(k-1) / 2\rceil+1}{\lceil(k-1) / 2\rceil}$. The result now follows from the trivial inequality $\operatorname{CRT}(k) \leqslant \mathrm{CRT}(k-1)$.

Remark 8. Lower bound from Proposition 1 works for $k=4$ and $k=5$ also, and the computer check for short lengths shows that these two cases are likely to satisfy the general formula. But at the moment we cannot prove the upper bound that meets the lower bound. Using the construction similar to the ones used for $k=6,7,9$, we can only show that $3 / 2 \leqslant \mathrm{CRT}(4) \leqslant \mathrm{RT}(3)=7 / 4$ and $4 / 3 \leqslant \mathrm{CRT}(5) \leqslant 3 / 2$. 

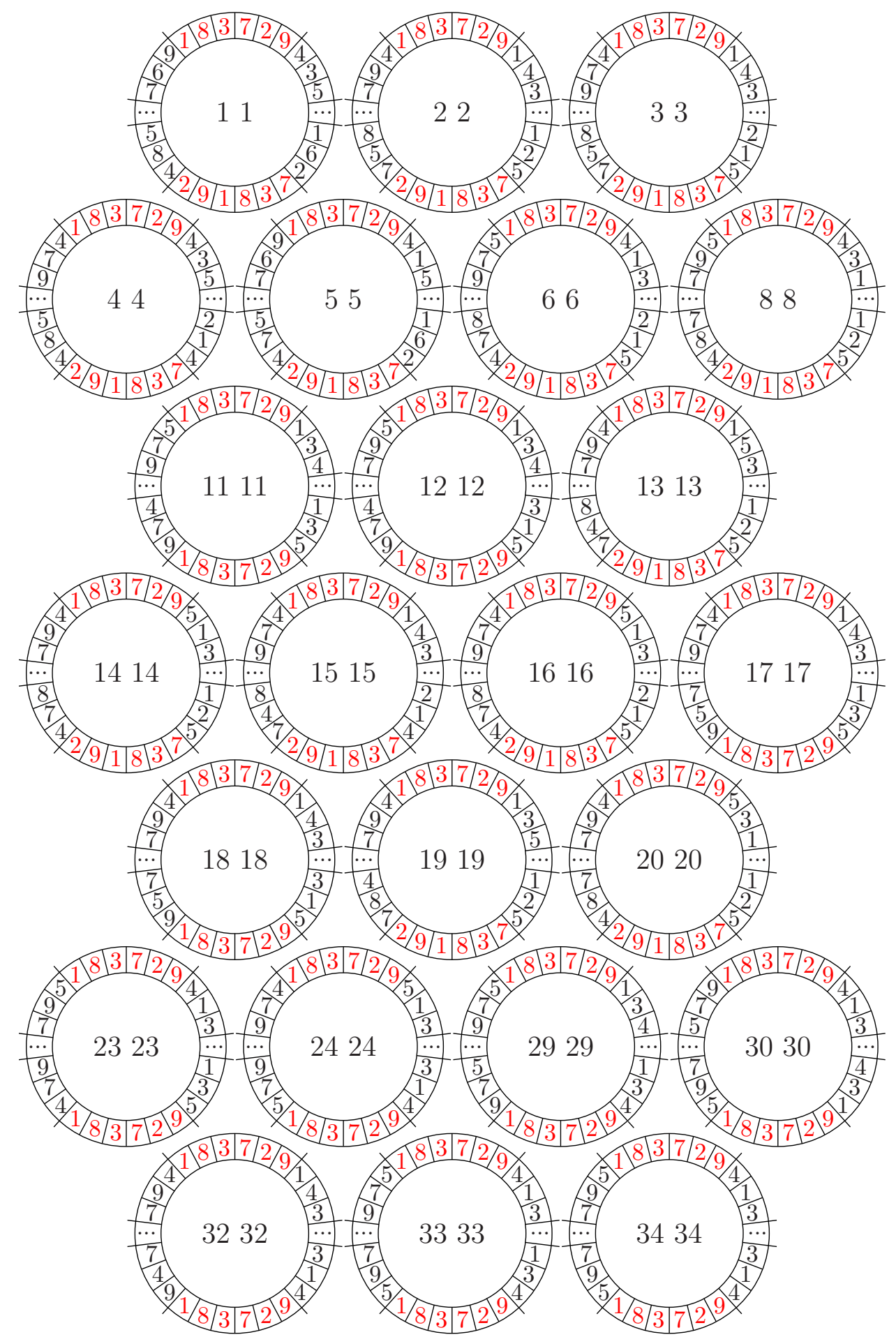

Figure 16: Circular (6/5)+-free words of length $n$ ( $n \geqslant 24$ is even) over 9 letters. The types of $u$ and $v$ are given in the center of the circle. 


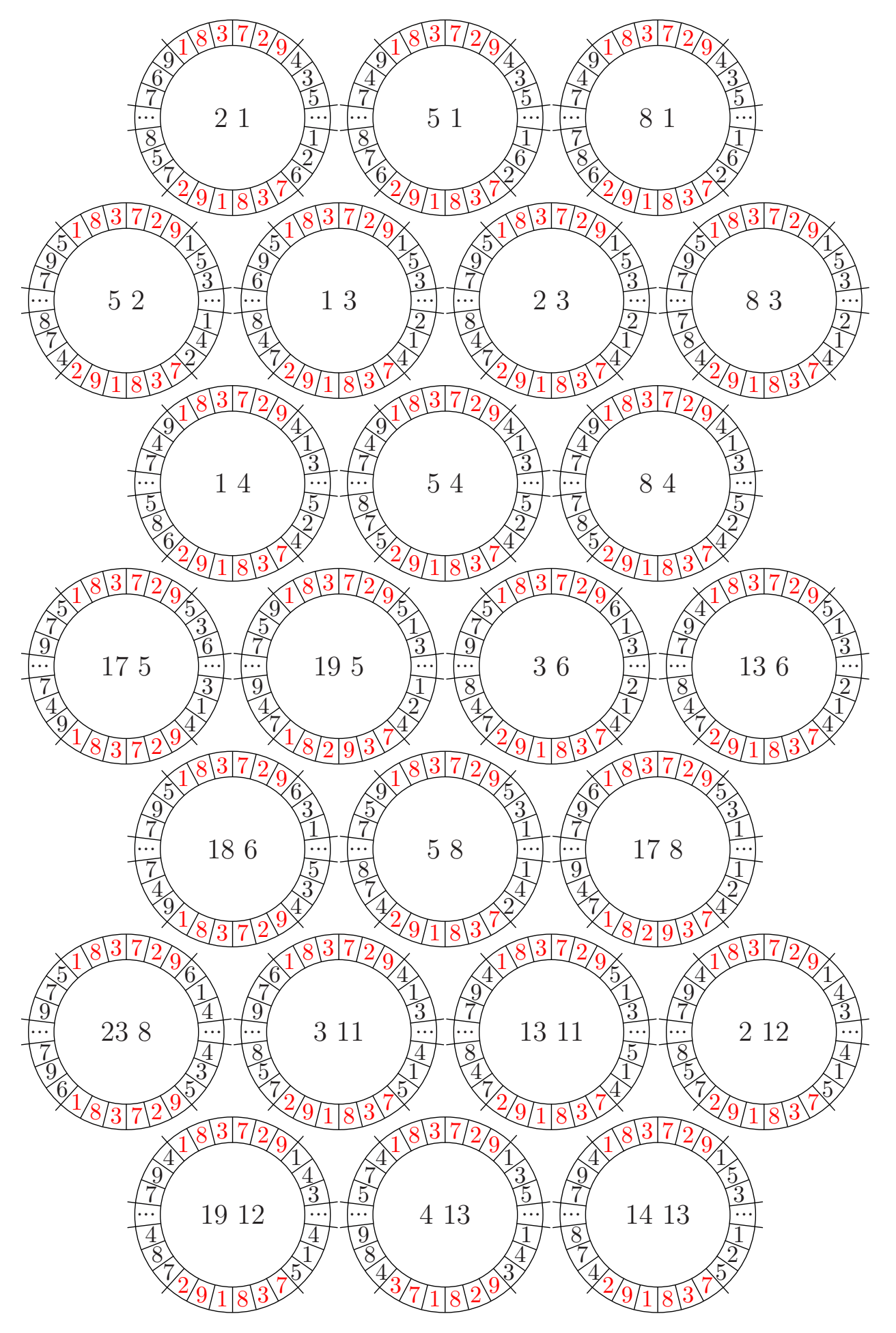

Figure 17: Circular (6/5)+-free words of length $n$ ( $n \geqslant 24$ is odd) over 9 letters. 

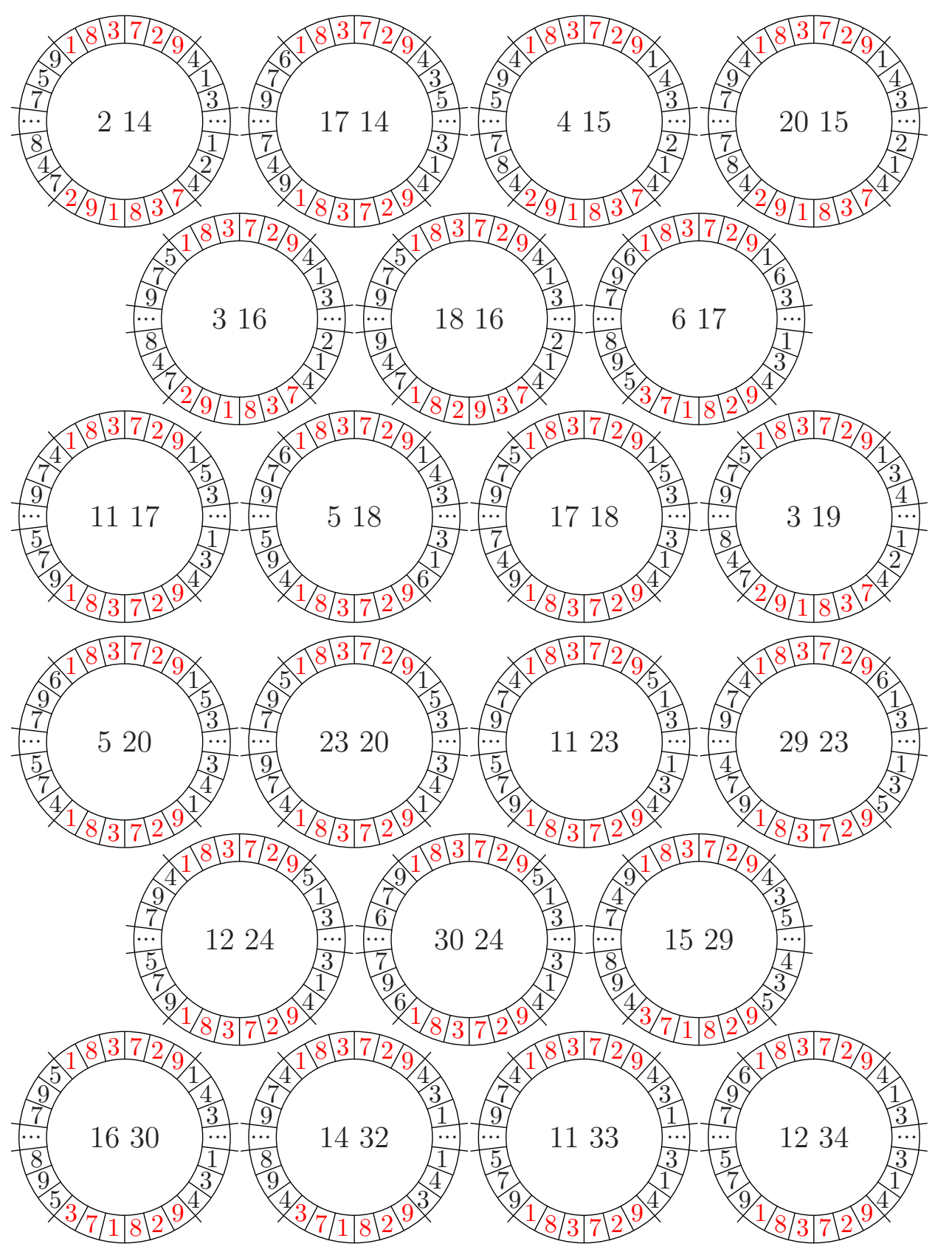

Figure 18: Circular $(6 / 5)^{+}$-free words of length $n$ ( $n \geqslant 24$ is odd) over 9 letters (continued). 
Thus, the circular repetition threshold $\mathrm{CRT}(k)$ is supposed to look as follows (only the cases $k=4,5$ are not proved, only the cases $k=2,3$ do not satisfy the general formula):

\begin{tabular}{|c|c|c|c|c|c|c|c|c|}
\hline$k$ & 2 & 3 & 4 & 5 & 6 & $\cdots$ & $m$ & $\cdots$ \\
\hline $\operatorname{CRT}(k)$ & $5 / 2$ & 2 & $3 / 2$ & $4 / 3$ & $4 / 3$ & $\cdots$ & $(\lceil m / 2\rceil+1) /\lceil m / 2\rceil$ & $\cdots$ \\
\hline
\end{tabular}

\section{Acknowledgements}

The author is grateful to Professor A.M. Shur for numerous suggestions that helped to greatly improve the paper.

\section{References}

[1] Ali Aberkane and James D. Currie. There exist binary circular $5 / 2^{+}$power free words of every length. Electr. J. Comb., 11(1), 2004.

[2] Arturo Carpi. On Dejean's conjecture over large alphabets. Theor. Comput. Sci., 385(1-3):137-151, 2007.

[3] James D. Currie and Narad Rampersad. A proof of Dejean's conjecture. Math. Comput., 80(274):1063-1070, 2011.

[4] Françoise Dejean. Sur un théorème de Thue. J. Comb. Theory, Ser. A, 13(1):90-99, 1972.

[5] Francesca Fiorenzi, Pascal Ochem, and Elise Vaslet. Bounds for the generalized repetition threshold. Theor. Comput. Sci., 412(27):2955-2963, 2011.

[6] Lucian Ilie, Pascal Ochem, and Jeffrey Shallit. A generalization of repetition threshold. Theor. Comput. Sci., 345(2-3):359-369, 2005.

[7] Michaël Rao. Last cases of Dejean's conjecture. Theor. Comput. Sci., 412(27):30103018, 2011.

[8] Andrey Rumyantsev. Upper bound for the generalized repetition threshold. 2010. arXiv: 1009.4454.

[9] Alexey V. Samsonov and Arseny M. Shur. On Abelian repetition threshold. RAIRO - Theor. Inf. and Applic., 46:147-163, 2012.

[10] Arseny M. Shur. On the existence of minimal $\beta$-powers. Int. J. Found. Comput. Sci., 22(7):1683-1696, 2011. 